\title{
Nonlinear rheology of polydisperse blends of entangled linear polymers: Rolie-Double- Poly models
}

Victor A. H. Boudara, Joseph D. Peterson, L. Gary Leal, and Daniel J. Read

Citation: Journal of Rheology 63, 71 (2019); doi: 10.1122/1.5052320

View online: https://doi.org/10.1122/1.5052320

View Table of Contents: https://sor.scitation.org/toc/jor/63/1

Published by the The Society of Rheology

\section{ARTICLES YOU MAY BE INTERESTED IN}

Near wall velocimetry on a rheometer

Journal of Rheology 63, 93 (2019); https://doi.org/10.1122/1.5047020

Review on tube model based constitutive equations for polydisperse linear and long-chain branched polymer melts

Journal of Rheology 63, 361 (2019); https://doi.org/10.1122/1.5064642

Letter to the Editor: Melt rupture unleashed by few chain scission events in fully stretched strands Journal of Rheology 63, 105 (2019); https://doi.org/10.1122/1.5054655

Polymer extrudate-swell: From monodisperse melts to polydispersity and flow-induced reduction in monomer friction

Journal of Rheology 63, 319 (2019); https://doi.org/10.1122/1.5058207

Modifying the rheological properties of polypropylene under elongational flow by adding polyethylene Journal of Rheology 63, 11 (2019); https://doi.org/10.1122/1.5049378

Relaxation time of dilute polymer solutions: A microfluidic approach

Journal of Rheology 61, 327 (2017); https://doi.org/10.1122/1.4975933

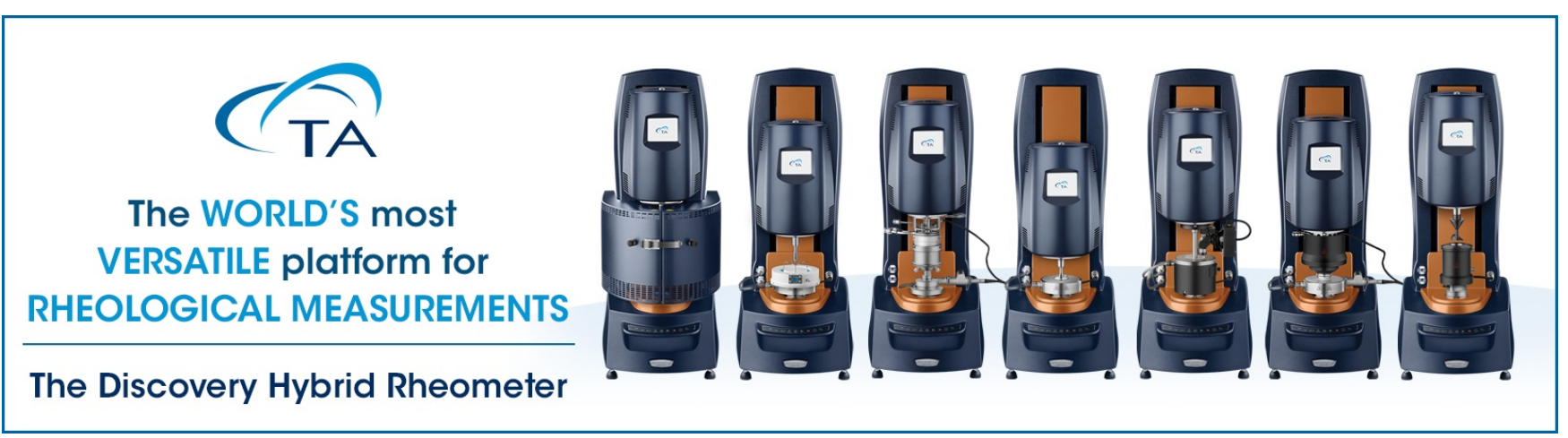




\title{
Nonlinear rheology of polydisperse blends of entangled linear polymers: Rolie-Double-Poly models
}

\author{
Victor A. H. Boudara, ${ }^{1}$ Joseph D. Peterson, ${ }^{2}$ L. Gary Leal, ${ }^{2}$ and Daniel J. $\operatorname{Read}^{1, a)}$ \\ ${ }^{1}$ School of Mathematics, University of Leeds, LS2 9JT Leeds, United Kingdom \\ ${ }^{2}$ Department of Chemical Engineering, University of California, Santa Barbara, California 93106
}

(Received 16 August 2018; final revision received 1 November 2018; published 3 December 2018)

\begin{abstract}
While there has been much success in modeling the linear and nonlinear rheology of monodisperse entangled linear polymers, progress in the constitutive modeling of polymeric materials continues to lag behind the needs of industry. Industrially sourced polymers are typically polydisperse (comprising a broad distribution of molecular weights), making their rheology more suitable for processing but also more difficult to predict. To date, there are no molecular-based constitutive models that are practically suitable for describing industrially relevant polymers in industrially relevant flows. In this article, we extend but strongly simplify the model of Read et al. [J. Rheol. 56, 823-873 (2012)], which is able to predict the linear and nonlinear rheology of bidisperse blends but is prohibitively complex for industrial use. We propose a pair of simplified tube models for polydisperse melts of entangled linear polymers that combine the success of the double reptation approximation [des Cloizeaux, Europhys. Lett. 5, 437-442 (1988)] in the linear regime with the success of the Rolie-Poly constitutive equation [Likhtman et al., J. Non Newtonian Fluid Mech. 114, 1-12 (2003)] in the nonlinear regime. We first review the key concepts of the double reptation approximation and the original (monodisperse) Rolie-Poly constitutive model. Subsequently, we provide the details of our approximate models for the particular case of a bidisperse blend and show that these models naturally identify the effects from couplings between constraint release and chain retraction (i.e., the so-called "enhanced stretch relaxation time"). Finally, we generalize to a multicomponent (polydisperse) model, based on the same underlying principles. Along the way, we also show that both of our models are in qualitative, and largely quantitative, agreement with experimental data for bidisperse and polydisperse melts of entangled linear polymers. (C) 2018 The Society of Rheology. https://doi.org/10.1122/1.5052320
\end{abstract}

\section{INTRODUCTION}

\section{A. Polydispersity in entangled polymers}

For academic study, it is possible to synthesize and isolate small quantities of nearly monodisperse polymers, but practically all industrial polymers are polydisperse, comprising a broad distribution of molecular weights. It is widely recognized that polydispersity, especially as it pertains to the longest chains, is an important factor in rheological and processing behavior and can have a significant influence on the transient and steady state response to the flow of polymeric systems [1-3].

Considerable progress has been made in describing, from a theoretical and practical point of view, the linear and nonlinear rheology of nearly monodisperse linear polymers [1]. Building on the early work of de Gennes [4,5] and Doi and Edwards [6], modern constitutive models (such as the so-called GLaMM (Graham, Likhtman and McLeish, Milner) model [7]) have been developed to incorporate fundamental molecular stress relaxation processes such as reptation, chain retraction, and convective constraint release (CCR) [8]. These models make quantitative predictions that are generally in good agreement with experimental data for monodisperse melts in strong (nonlinear) flow conditions [9]. Furthermore,

\footnotetext{
a) Author to whom correspondence should be addressed; electronic mail: d.j.read@leeds.ac.uk
}

simplified "toy" models, such as the Rolie-Poly model [10], retain the main features of more detailed models but permit computation in complex nonrheometric flows [2,11-13].

By contrast, the theoretical predictions of the modern versions of Doi-Edwards theory [6], such as GLaMM [7], fail to capture even the linear rheology of polydisperse melts. The Doi-Edwards framework coarse grains the set of constraints (entanglements) acting on a polymer into a fixed tubelike confining potential. Approximating constraints as fixed is a reasonable first approximation for monodisperse systems, but for melts of very long and very short chains, the impermanence of the short chain constraints can no longer be neglected. As short chains rapidly diffuse within the melt, they facilitate a thermally driven release and renewal of entanglements called "constraint release" (CR) for short. With each CR event, the long chains (which are practically not diffusing by comparison) are able to partially renew their configuration and relax a portion of their stress. There is a superficial simplicity to $\mathrm{CR}$, but upon closer inspection, the details become so complex that a completely general, rigorous, theory has not yet been developed.

Building on (and to an extent simplifying) the idea of Rubinstein and Colby [14], the double reptation approximation of des Cloizeaux [15] outlines a simple and intuitive strategy for improving upon the predictions of reptation theory without introducing additional free parameters. For a wide range of blend compositions, this semiempirical description of $\mathrm{CR}$ is reasonably accurate for predictions of 
linear viscoelasticity [1]. However, as we will discuss in more detail below, double reptation often is an imperfect approximation for some aspects of stress relaxation in binary blends. For example, in many bidisperse blends, the terminal relaxation time of long chains is decreased by the presence of short chains (often this acceleration of long chain relaxation is clearly visible in the linear rheology data irrespective of the method used to extract the terminal relaxation time; a survey of the literature data can be found in [16]). The acceleration of long chains by short chains is not naturally accounted for in a double reptation model $[1,16]$. Therefore, we employ the double reptation formalism as a starting point but allow for physically motivated corrections as needed.

\section{B. Description of the double reptation approximation}

Double reptation describes polymer blends through a semiempirical temporary network approximation. The full complexities of topological interactions between chains are coarse-grained into isolated, binary entanglements acting as temporary cross-links. These cross-links accumulate stress during deformation and relax stress only when the cross-link is removed at some later time.

Assuming entanglements form between two chain segments, with a probability that is independent of the molecular weight of the attached chains, then for any given test chain $i$, the fraction of the entanglements on that test chain coming from chains of type $j$ is just $\phi_{j}$, the volume fraction of the $j$ chains. But, test chains of type $i$ are present with volume fraction $\phi_{i}$, hence the fraction of $i-j$ entanglements (i.e., entanglements on chain type $i$ coming from chain type $j$ ) is $\phi_{i} \phi_{j}$, and likewise the fraction of $j-i$ entanglements is $\phi_{j} \phi_{i}$. Overall, the total fraction of entanglements of either $i-j$ or $j-i$ (i.e., involving distinct species) is $2 \phi_{i} \phi_{j}$, and the total fraction of entanglements of type $i-i$ (i.e., involving identical species) is just $\phi_{i} \phi_{i}$.

The rate of cross-link release is dictated by tube model descriptions of the relaxation kinetics for the constituent monodisperse fractions within the melt. The two chains may disengage from one another when either of the two chains reptates past the relevant portion of its effective "tube." We can define $p_{i}(t)$ as the probability that a randomly selected entanglement segment from chains of type $i$ has not been passed by the $i$-chain end, via reptation or contour length fluctuation (CLF), after some time $t$ [i.e., $p_{i}(t)$ is the tube survival probability for chains of type $i]$. Assuming that the reptation of $i$ and $j$ are independent events, then the survival probability $p_{i j}(t)$ of an $i$-j entanglement is just the product of the individual tube survival probabilities of the two chains, i.e., $p_{i j}(t)=p_{i}(t) p_{j}(t)$. To a first approximation, $p_{i}(t)$ and $p_{j}(t)$ can be estimated from the fixed-tube reptation kinetics of each chain, i.e., a usual assumption of the double reptation theory is that the relaxation function $p_{i}(t)$ of species $i$ is unaffected by the presence, or otherwise, of species $j$.

Double reptation further assumes that the stress relaxation function following a step strain is proportional to the fraction of remaining entanglements after time $t$, i.e., it considers the entanglements effectively to be the stress-inducing objects.
Hence, the contribution to the stress relaxation modulus associated with $i$-j entanglements is $G_{i j}(t)=G_{N}^{0} \phi_{i} \phi_{j} p_{i j}(t)$, where the plateau modulus, $G_{N}^{0}$, is typically considered independent of the molecular weight distribution, and (consistently with the entanglement counting above) we count $i-j$ entanglements and $j-i$ entanglements separately. If there are $n$ components altogether, the overall relaxation modulus $G(t)$ is given by

$$
G(t)=\sum_{i=1}^{n} \sum_{j=1}^{n} G_{i j}(t)
$$

Double reptation is most commonly applied for understanding the linear viscoelastic properties of a polymer melt, but in principle $G(t)$ could be inserted into an integral constitutive equation to obtain rheological predictions under strong flow conditions as well [17]. As a simple example, we can use a Maxwell model

$$
\boldsymbol{\sigma}(t)=\int_{-\infty}^{t} \frac{\partial}{\partial t} G\left(t-t^{\prime}\right) \mathbf{C}^{-1}\left(t, t^{\prime}\right) \mathrm{d} t^{\prime},
$$

where $\boldsymbol{C}^{-1}\left(t, t^{\prime}\right)$ is the finger tensor representing affine deformation occurring between times $t$ and $t^{\prime}$. For any material with single-mode relaxation kinetics, i.e., $p_{i}(t) \propto \exp \left(-t / \tau_{\mathrm{d}, i}\right)$, this integral Maxwell model has a related differential form

$$
\left\{\begin{array}{l}
\boldsymbol{\sigma}(t)=\sum_{i=1}^{n} \sum_{j=1}^{n} \phi_{i} \phi_{j} \boldsymbol{\sigma}_{i j}(t) \\
\nabla_{i j}=-\left(\frac{1}{\tau_{\mathrm{d}, i}}+\frac{1}{\tau_{\mathrm{d}, j}}\right)\left(\boldsymbol{\sigma}_{i j}-G_{N}^{0} \boldsymbol{I}\right)
\end{array}\right.
$$

where

$$
\stackrel{\nabla}{\boldsymbol{A}} \equiv \frac{\mathrm{D} \boldsymbol{A}}{\mathrm{D} t}-\left(\boldsymbol{\kappa} \cdot \boldsymbol{A}+\boldsymbol{A} \cdot \boldsymbol{\kappa}^{T}\right)
$$

is the upper convected derivative representing the effect of affine deformation on a tensor $\boldsymbol{A}$, and $\boldsymbol{\kappa}$ is the velocity gradient tensor.

When the double reptation approximation is presented in such a differential constitutive form, $\boldsymbol{\sigma}_{i j}$ can be interpreted as the stress contribution arising from chain $i$ due to entanglements with chain $j$. We may also note that whereas independent modes of relaxation are multiplicative when computing $G_{i j}(t)$, the corresponding relaxation rates are additive when computing the evolution of $\boldsymbol{\sigma}_{i j}$.

In the present report, we will pursue a generalization (or extension) of the double reptation approximation, building upon this differential constitutive framework for double reptation and incorporating effects of CLFs, chain retraction, and CCR.

\section{Beyond published double reptation models}

Differential constitutive models incorporating ideas from double-reptation have been around for over two decades and were probably developed independently on more than one 
occasion. The earliest example of such work comes from Marrucci and Ianniruberto [18,19], but as far as we can tell, their model was never formally used to study polydisperse systems. A closely related model by Mead [20] and its extensions [21-24] incorporate similar ideas and are more explicitly intended for polydisperse systems. In particular, Mead et al. [24] outlines a nonlinear rheology model for polydisperse systems where the description of the stretch is done via elaborate bookkeeping measures, counting discrete $i j$ entanglement pairs on the chain when entanglement dynamics and stretch tube dilution operate. This work also incorporates the possibility of flow-induced reduction in entanglement density.

A potential weakness of these existing works is that stretch and orientation dynamics are treated using separate dynamical equations, which can lead to problems even in the single-mode (monodisperse) rheology predictions [25]. Here, we argue that this decoupling approximation is even more problematic for polydisperse blends; couplings between CR and chain retraction (related to the "enhanced Rouse time" phenomena $[9,26]$ ) must be readmitted in an ad hoc fashion and do not arise naturally from the model [23]. In contrast, our work described below follows the GLaMM and Rolie-Poly models [7,10], keeping the stretch and orientation dynamics in a single tensor equation: The result is a very natural generalization of these two models and of the results of Auhl et al. [9,26] for bidisperse polymers. The stretch dynamics, in particular, the "enhanced Rouse time," emerge directly from our equations and do not appear to require the elaborate bookkeeping of stretch dynamics [24].

Finally, in a seemingly unrelated vein of research, Jupp and Yuan [27,28] developed an approach in which some double reptation concepts were adapted to preexisting constitutive models (Johnson-Segalman and Rolie-Poly). These models do not decouple stretch and orientation, but they lack internal consistency (Rolie-Poly) or are too phenomenological (Johnson-Segalman) to be reliable for qualitative or quantitative rheological predictions.

\section{Limitations of the double reptation approximation-Viovy diagram}

Two of the common assumptions of double reptation theory, as described above, may be summarized as follows: (i) that entanglements are binary events, so the contribution to the modulus from entanglements of chains $i$ with chains $j$ is proportional to $\phi_{i} \phi_{j}$ and (ii) that the relaxation function $p_{i}(t)$ of species $i$ is unaffected by the presence of species $j$. For the long chains in a binary blend, the consequences of these two assumptions are that (i) the terminal modulus associated with the long chains should be proportional to the square of their volume fraction, $\phi_{\mathrm{L}}^{2}$ and (ii) their terminal relaxation time should be unaffected by dilution with short chains. Unfortunately, in many cases, one, or both, of these assumptions fail when compared with experimental data, as we explain below $[1,16,26]$. In contrast, for fully polydisperse systems many of the detailed dynamics are hidden by the broad distribution of relaxation times, and double reptation is often quite successful [1].

A more detailed appreciation of the regimes of applicability of double reptation for binary blends of entangled polymers can be attained through consideration of the "Viovy diagram" [29], shown schematically in Fig. 1(a), and its recent modification by Read et al. [16] shown schematically in Fig. 1(b). The diagram is a two-dimensional projection of the (three dimensional) binary blend parameter space. The horizontal axis is the typical number of entanglements a long chain has with other long chains. The vertical axis is the a)

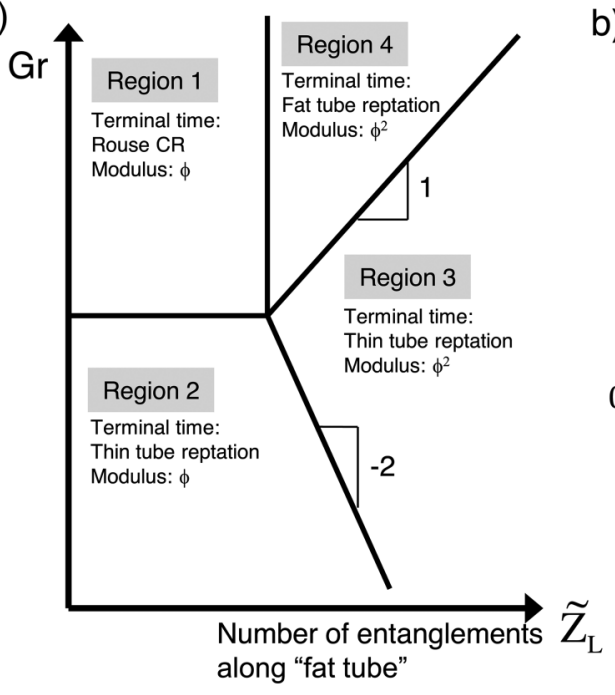

b)

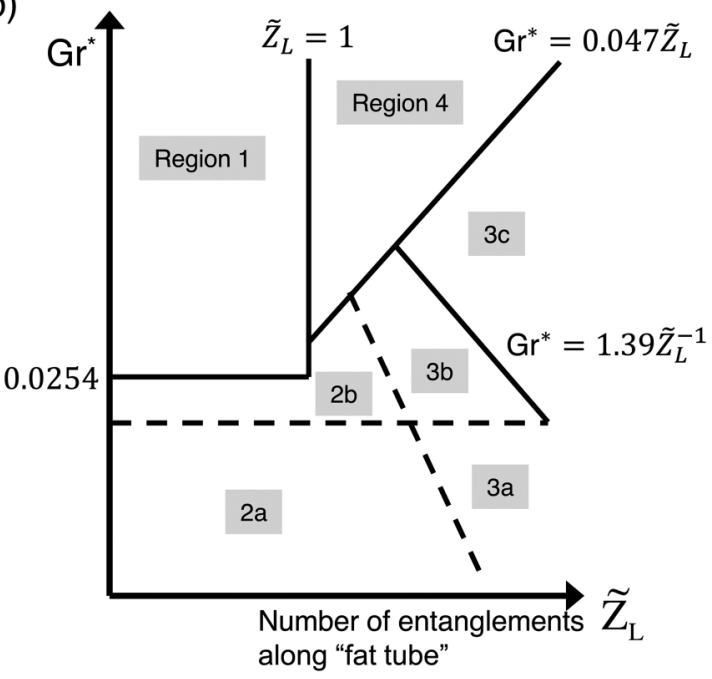

FIG. 1. (a) Schematic Viovy diagram [29] indicating, in each region, the process governing terminal relaxation and how the terminal modulus scales with fraction $\phi$ of long chains. In region 1 , dilute long chains relax terminally through CR; in region 2 , they relax by reptation. In regions 3 and 4 , long chains are selfentangled hence the terminal modulus scales as $\phi^{2}$; in region 3, reptation along the thin tube dominates, while in region 4, motion along the fat tube, occurring due to CR of the thin tube, is predicted to accelerate the terminal reptation of the long chains. (b) Modified Viovy diagram as obtained by Read et al. [16] including the effects of CLF. Region 3 is divided into three subregions: $3 a$, only CLF along the thin tube occurs; $3 b$, CLF occurs in a tube diluting by CR Rouse motion; and 3c, CLF occurs in the fat tube. Region 2 is also subdivided into: $2 \mathrm{a}$, only CLF along the thin tube occurs and $2 \mathrm{~b}$, CLF occurs in a tube diluting by CR Rouse motion. Equations for some lines indicated as derived from slip-spring models. 
Graessley parameter, $\mathrm{Gr}=Z_{\mathrm{L}} / Z_{\mathrm{S}}{ }^{3}$ [1], and is a measure of the relative rate of long chain relaxation by $\mathrm{CR}$ from short chains, as compared to reptation (higher values of Gr represent faster CR). It is implicit in the construction of the diagram that the short chains are somewhat shorter than the long ones, i.e., $Z_{\mathrm{S}}<Z_{\mathrm{L}}$, meaning also that $\mathrm{Gr}>1 / Z_{\mathrm{L}}{ }^{2}$. The diagram is divided into a number of regions according to the expected relaxation pathway of the long chains. The original diagram [Fig. 1(a)] considered only the effects of reptation and CR: This gives a perfect projection into the two dimensional space of the diagram. The modified diagram [Fig. 1(b)] includes additionally the effects of CLFs; as a result, some of the lines on the diagram, shown with dashed lines, move weakly with the third independent parameter (for which there are many choices, but which can conveniently be taken as the undiluted entanglement number $Z_{\mathrm{L}}$ ).

To the right of the diagram (regions 3 and 4), long chains feel the effects of entanglements with other long chains before terminal relaxation. Hence, the terminal modulus from long chains scales as $G_{N}^{0} \phi_{\mathrm{L}}^{2}$, where $G_{N}^{0}$ is the plateau modulus, in agreement with double reptation. In contrast, to the left of the diagram (regions 1 and 2), the long chains are too dilute to entangle with other long chains, and so the terminal modulus is simply proportional to the long chain concentration $\phi_{\mathrm{L}}$. Hence, the prediction of double reptation for terminal modulus fails to the left of the Viovy diagram.

Toward the lower end of the Viovy diagram, CR from the short chains is sufficiently slow that the fastest mechanism to relax long chains remains reptation along their undiluted tube. Hence, in regions 2 and 3 of the original Viovy diagram, the predictions of double reptation for the relaxation time of the long chains are expected to hold. Toward the top of the diagram, CR from short chains accelerates the relaxation of the long chains, either through CR Rouse relaxation of long chains (region 1) or motion of long chains along the "fat tube" of other long chain entanglements, at an accelerated rate determined by $\mathrm{CR}$ from the short chains (region 4). In these two regions, short chains affect the long chain relaxation rate and so the prediction of double reptation for terminal relaxation time fails. This situation becomes yet more complicated in the modified diagram [Fig. 1(b)] of Read et al. [16] who identified that CLFs in the diluted fat tube provide a further acceleration mechanism for long chain relaxation in much of region 3 (specifically, regions $3 b$ and $3 c$ ): Hence, the double reptation prediction of long chain relaxation time fails also for a significant part of region 3 !

As a consequence, double reptation in its unmodified form is technically valid only in a small part of the parameter space [region 3a of Fig. 1(b)] since only here are the predictions of both terminal time and terminal modulus for the long chains valid. However, to the right of the Viovy diagram, a modified double reptation scheme can be successful, provided that one modifies the relaxation time of long chains to account for the presence of short chains in the surrounding medium. In this paper, we will introduce appropriate (but approximate) schemes to achieve this prediction of relaxation times, while also retaining the $\phi_{i} \phi_{j}$ scaling for the modulus contribution from entanglements on chains $i$ from chains $j$. Therefore, the constitutive model presented here could be applied throughout regions 3 and 4 of the Viovy diagram.

\section{E. Outline of this work}

The central purpose of this paper is to develop a nonlinear constitutive model for polydisperse linear entangled polymers that is (i) simple enough for computational fluid dynamics (CFD) studies and (ii) accurate enough to deal with high polydispersity and high deformation rates.

We will present two constitutive models, satisfying objectives (i) and (ii) above, that we call "Rolie-Double-Poly" (RDP) and "Symmetric Rolie-Double-Poly" (SRDP), where the "Double" signifies both "double reptation" and "double poly" as in "POLYdisperse POLYmers." Although these models were originally constructed independently, from different microscopic pictures, their conceptual, structural, and quantitative similarity is the reason why we have elected to present them in a single publication.

In Sec. II, we study the specific case of a bidisperse blend of long and short chains. We start with a short review of the monodisperse Rolie-Poly model [10]. Subsequently, in Sec. II B, we define our "RDP" and "SRDP" models for the case of bidisperse blends and summarize our present understanding for the quantitative agreement between the two formulations. One important qualitative similarity, discussed in Sec. II $\mathrm{C}$, is that both models exhibit the natural emergence of an enhanced stretch relaxation time [26,30], arising from the coupling between stretch relaxation and CR processes. Given that the two models are quantitatively indistinguishable for cases where experimental data are available, in the interest of concise exposition, calculations in the main body of the paper and comparisons to data will only be presented for the RDP model. We compare the model to experimental linear and nonlinear rheology data for bidisperse melts in shear and extension. For qualitative purposes, the RDP (and SRDP) model requires no free parameters, but for quantitative interests, we require a set of experimentally tabulated tube-model parameters (molecular weight between entanglements $M_{\mathrm{e}}$, Rouse time of an entanglement strand $\tau_{\mathrm{e}}$, and plateau modulus $G_{N}^{0}$ ) to specify the model. In principle, $M_{\mathrm{e}}$ and $G_{N}^{0}$ are not independent parameters but are linked through Eq. (44); in practical application, these are often treated as independent parameters with Eq. (44) only approximately enforced. We further allow a single fitting parameter to correct for departures of the terminal relaxation modulus of the blends from predictions of double reptation based solely on total weight fraction of long chains. Such departures are particularly acute in binary blends, and they arise because some sections of the long chains (i.e., chain ends) relax relatively rapidly and provide extra dilution to the terminal modulus. This fitting parameter is set by the linear rheology alone so that predictions of the nonlinear rheology (shear and extension) are made without any further intervention.

Finally, in Sec. III, we generalize the bidisperse RDP and SRDP models to the general polydisperse case. Again, given the quantitative agreement between RDP and SRDP, we compare the resulting predictions with experimental 
observations of linear and nonlinear rheology in polydisperse polymers for the RDP model only. In the linear regime, the model yields quantitatively accurate predictions without any adjustable parameters or empirical correlations; once the molecular weight distribution is specified, no further free parameters are needed to define the model. This result is achieved through a novel strategy for approximating the effect of couplings between CR and CLFs in polydisperse melts developed in the Appendix. In the nonlinear regime, initial comparisons with nonlinear rheological data of polydisperse polymers, presented in Sec. III B, indicate that the nonlinear features are captured with good accuracy as well. Hence, both the linear and nonlinear rheology can be predicted from this molecular-based theory, based only on a set of experimentally tabulated tube-model parameters.

\section{NONLINEAR RHEOLOGY OF BIDISPERSE BLENDS}

\section{A. Preamble: The "classic" Rolie-Poly model for monodisperse polymers}

\section{Formulation of the Rolie-Poly model}

Before delving into the blend model formulations, we first take a moment to review the classic Rolie-Poly model of Likhtman and Graham [10], which has its origins in the full chain GLaMM model.

The GLaMM model was originally proposed by Graham and co-workers [7] as a full chain molecular theory for entangled monodisperse linear polymer chains under fast deformation. The GLaMM model accounts for an accumulation of stress via affine deformation and relaxation of stress by reptation, chain retraction, and CCR - as introduced by Marrucci [31]. Although successful in predicting the rheology of fast flows, the GLaMM model is computationally prohibitive for nonviscometric flow calculations; the dimensionality of the model is high (three spatial dimensions plus two chain contour indices) and the dynamics are stiff (the model includes all relaxation times down to $\tau_{\mathrm{e}}$ ). Hence, as a simplified version of the GLaMM model, Likhtman and Graham developed a one-mode differential constitutive equation for entangled monodisperse polymer chains called the Rolie-Poly equation (for Rouse linear entangled polymers) [10]. The time evolution of the stress tensor of the polymer melt, $\boldsymbol{\sigma}$, is calculated via a conformation tensor for an entanglement segment, $\boldsymbol{A}$, as

$$
\left\{\begin{aligned}
\boldsymbol{\sigma}(t) & =G_{N}^{0} \mathrm{f}_{\mathrm{E}}(\lambda) \boldsymbol{A}(t) \\
\nabla & \\
\boldsymbol{A} & =-\boldsymbol{R}_{\mathrm{rep}}-\boldsymbol{R}_{\mathrm{CR}}-\boldsymbol{R}_{\mathrm{ret}}-\boldsymbol{R}_{\mathrm{CCR}}
\end{aligned}\right.
$$

where the stretch ratio of the polymer chains, i.e., the ratio of the current to equilibrium-with-no-flow chain contour lengths, is defined as $\lambda=(\operatorname{Tr} \boldsymbol{A} / 3)^{1 / 2}$ and with the reptation (rep), CR, retraction (ret), and CCR relaxation terms defined, respectively, as

$$
\boldsymbol{R}_{\text {rep }} \equiv \frac{1}{\tau_{\mathrm{d}}}(\boldsymbol{A}-\boldsymbol{I}),
$$

$$
\begin{gathered}
\boldsymbol{R}_{\mathrm{CR}} \equiv \frac{\beta_{\mathrm{th}}}{\tau_{\mathrm{d}}}(\boldsymbol{A}-\boldsymbol{I}), \\
\boldsymbol{R}_{\mathrm{ret}} \equiv \frac{2\left(1-\lambda^{-1}\right)}{\tau_{\mathrm{s}}} \mathrm{f}_{\mathrm{E}}(\lambda) \boldsymbol{A}, \\
\boldsymbol{R}_{\mathrm{CCR}} \equiv \beta_{\mathrm{CCR}} \frac{2\left(1-\lambda^{-1}\right)}{\tau_{\mathrm{s}}} \mathrm{f}_{\mathrm{E}}(\lambda) \lambda^{2 \delta}(\boldsymbol{A}-\boldsymbol{I}) .
\end{gathered}
$$

The time scale $\tau_{\mathrm{d}}$ is the "disengagement time," i.e., the longest time scale for the chain to escape from a fixed tube by the combined action of CLFs and reptation. $\tau_{\mathrm{s}}$ is the stretch relaxation time, which is anticipated to equal the Rouse time of the chain: to relax stretch (along tube) in a single entanglement segment, the whole chain needs to retract along the tube, so, to good approximation, the stretch relaxation time is the Rouse time of the whole chain. Similarly, to relax orientation of the entanglement segment, the whole chain needs to reptate. $\left(\beta_{\mathrm{CCR}}, \delta\right)$ are the CCR coefficients taken equal to $(1,-1 / 2)$, and $\beta_{\text {th }}$ is the thermal CR coefficient taken equal to 1 . It is the correct value to choose to be consistent with double reptation, although, in principle, stress relaxation via tube escape and CR could have different weightings. Note that we decorated the original Rolie-Poly model with finite extensibility [32], via the Warner approximation [33] of the inverse Langevin function

$$
\mathrm{f}_{\mathrm{E}}(\lambda) \equiv \frac{1-\lambda_{\max }^{-2}}{1-\lambda^{2} \lambda_{\max }^{-2}}
$$

with $\lambda_{\max }$ the maximum stretch ratio. For the rest of this work, we take $\lambda_{\max }=10$, unless stated otherwise. This parameter usually depends on the chemistry and on dilution, but it does not really affect the model predictions presented in this work in shear. In elongation, when elongation hardening would lead to diverging stress in the Rolie-Poly model, this parameter enforces a finite steady state stress value.

At first glance, our introduction of thermal CR, Eq. (7), appears to disagree with the usual presentation of the monodisperse Rolie-Poly model, where such a term is not present. However, we argue that the difference is purely semantic. In the original Rolie-Poly model, $\tau_{\mathrm{d}}$ is the terminal stress relaxation time of the melt, whereas in our models, $\tau_{\mathrm{d}}$ is the "bare" disengagement time for a fixed tube (reptation time shortened by CLF, as described later). The difference is subtle but important; for monodisperse entangled polymer systems, the terminal stress relaxation time is often equated with a reptation time, but strictly speaking, the terminal stress relaxation time comprises a combination of reptation, CLFs, and thermal CR. So, typically, there is roughly a factor of 2 (or more) difference between the terminal stress relaxation time and the disengagement time [1]. In the above, we have explicitly separated out the effects of disengagement [reptation and CLF, Eq. (6)] from CR [Eq. (7)], in a manner which is both consistent with double reptation and helpful for our later presentation of the RDP and SRDP models.

On the CCR term, the factor of $\lambda_{\mathrm{S}}^{2 \delta}$ is a phenomenological correction introduced to improve fitting of rheological data at 
high shear rates. Loosely speaking, this fitting function can be interpreted via the GLaMM model, which predicts that more $\mathrm{CR}$ events are required to relax a chain that is stretched. This correction could also be applied to the thermal CR terms, although this is not done in the original Rolie-Poly model, and we regard this as an unnecessary complication: We are considering cases where well-entangled chains dominate the rheological response, and for these thermal CR is only significant relative to $\mathrm{CCR}$ when chains are not stretched.

\section{Wrong way toward polydispersity}

Given that the Rolie-Poly model offers a reasonable description for the rheology of monodisperse blends, a common way of trying to model bidisperse or polydisperse systems is to approximate the blend as a linear superposition of its monodisperse fractions (i.e., neglecting any microscopic couplings between chains of different lengths). For a melt containing $n$ components, the most straightforward way to define the total stress $\boldsymbol{\sigma}$ is as a sum over the component stresses:

$$
\boldsymbol{\sigma}=\sum_{i=1}^{n} \phi_{i} \boldsymbol{\sigma}_{i}
$$

where $\phi_{i}$ is the volume fraction of chain $i$ and $\boldsymbol{\sigma}_{i}$ is the average stress on chain $i$, as determined by the monodisperse Rolie-Poly equation for that component. This can actually be a good first approximation for blends that fall into region 2 of the Viovy diagram, where microscopic couplings between chains can be neglected to a first approximation. More generally, however, a linear superposition Rolie-Poly model (LSRP) is less useful; for high polydispersity blends, the failure of a LSRP model is evident even in its linear viscoelastic predictions!

Therefore, it is more common to separate linear superposition models from their microscopic interpretations, treating the volume fractions and relaxation times as adjustable parameters through which the model can be fit to linear and nonlinear rheological data. Unfortunately, after fitting the model to a specific set of rheological tests, there is no guarantee that its predictions will be transferable (useful under substantially different flow conditions). Furthermore, since the adjustable parameters have no physical interpretation, it is impossible to predict how the material parameters will change with the melt formulation.

In Secs. II B-II D, we outline the RDP and SRDP polymer blend models which overcome the weaknesses of LSRP by combining the best insights of double reptation and Rolie-Poly. As an improvement over LSRP, these models attempt to explicitly incorporate both linear and nonlinear microscopic couplings between chains. Even without any adjustable parameters, this yields qualitatively accurate and (apparently) transferable predictions for both the linear and nonlinear rheology of polydisperse melts.

\section{B. Description of the RDP and SRDP models for bidisperse melts}

In this section, we present the structure of the RDP and SRDP constitutive models for bidisperse melts of linear, well-entangled polymers. As we shall see, the two models primarily differ with regard to the symmetry (or asymmetry) of stress relaxation across entanglements between different types of chains. With regard to predictions of linear viscoelasticity, both models are consistent with the usual results of double reptation theory. The first model that we present is referred to as the "RDP model" for "Rolie-Double-Poly" (ROuse LInear Entangled Double reptation Polydisperse Polymers) where "Double" signifies both "double reptation," and "double poly" as in "POLYdisperse POLYmers." The RDP model can be understood as a greatly simplified version of the detailed molecular theory for bidisperse polymer melts of Read and co-workers [26], as we explicitly show in the supplementary material [51].

The second constitutive model, apparently more "ad hoc," applies the double reptation ansatz (and the entire micromechanical picture of a temporary network) to all nonlinear relaxation mechanisms of the Rolie-Poly model. As we shall see, this model is effectively a symmetrized version of the RDP model, we refer to this work as the "SRDP model" for "Symmetric-RDP" model, where enforcing symmetry is found to be quantitatively harmless but advantageous for the simulation of complex flows, as we will discuss later.

Note that a model for bidisperse blends of the forms presented here was circulating during the U.K. based Microscale Polymer Processing 2 project $\left(\mu \mathrm{PP}^{2}\right)^{1}$ and was partially encoded in RepTate software, ${ }^{2}$ but never published.

Beginning with a bidisperse melt, we denote quantities related to the shorter and longer chains with subscripts " $\mathrm{S}$ " and "L," respectively. We consider that our blend contains a volume fraction $\phi_{\mathrm{S}}$ of short chains, and a volume fraction $\phi_{\mathrm{L}}$ of long chains. We write the total stress tensor as

$$
\boldsymbol{\sigma}(t)=G_{N}^{0}\left(\phi_{\mathrm{S}} \mathrm{f}_{\mathrm{E}}\left(\lambda_{\mathrm{S}}\right) \boldsymbol{A}_{\mathrm{S}}(t)+\phi_{\mathrm{L}} \mathrm{f}_{\mathrm{E}}\left(\lambda_{\mathrm{L}}\right) \boldsymbol{A}_{\mathrm{L}}(t)\right),
$$

where $\boldsymbol{A}_{\mathrm{S}}$ and $\boldsymbol{A}_{\mathrm{L}}$ are the average conformation tensors of an entanglement segment in the short and long chains, respectively, and

$$
\lambda_{\mathrm{S}}=\left(\operatorname{Tr} \boldsymbol{A}_{\mathrm{S}} / 3\right)^{1 / 2}, \quad \lambda_{\mathrm{L}}=\left(\operatorname{Tr} \boldsymbol{A}_{\mathrm{L}} / 3\right)^{1 / 2},
$$

are the respective stretch ratios of the short and long chains.

The short chains and long chains entangle with other short and long chains, releasing their entanglements on different time scales. Therefore, we break down the conformation tensors $\boldsymbol{A}_{\mathrm{S}}$ and $\boldsymbol{A}_{\mathrm{L}}$ as

$$
\begin{aligned}
& A_{\mathrm{S}}=\phi_{\mathrm{S}} \boldsymbol{A}_{\mathrm{SS}}+\phi_{\mathrm{L}} \boldsymbol{A}_{\mathrm{SL}} \\
& \boldsymbol{A}_{\mathrm{L}}=\phi_{\mathrm{L}} \boldsymbol{A}_{\mathrm{LL}}+\phi_{\mathrm{S}} \boldsymbol{A}_{\mathrm{LS}}
\end{aligned}
$$

The above structure, in which stress is first separated out into chain contributions (via $\boldsymbol{A}_{\mathrm{S}}$ and $\boldsymbol{A}_{\mathrm{L}}$ ) and then

\footnotetext{
${ }^{1}$ http://www1.irc.leeds.ac.uk/mupp2/.

2“Old" RepTate can be found in http://reptate.com. A new version is available in http://reptate.readthedocs.io.
} 
subsequently separated out into contributions from chain pairs (e.g., $\boldsymbol{A}_{\mathrm{LL}}$ and $\boldsymbol{A}_{\mathrm{LS}}$ ) as in Eqs. (14) and (15), can be shown to be consistent with the much more detailed structure of correlation functions in the model of Read and co-workers [26]. We show this explicitly in the supplementary material [51]. In this interpretation, the tensor $\boldsymbol{A}_{I J}$ accounts for the effect of entanglements from chains of type $J$ on the average configuration for a tube segment of chains of type $I$ [where $I$ and $J$ could be short (S) or long (L)]. The RDP model preserves this interpretation.

Further (and for the long chains especially), we show in the supplementary material [51] that the tensor $\boldsymbol{A}_{\mathrm{L}}$ may be considered to be the configuration tensor for a "thin tube" entanglement segment, while $\boldsymbol{A}_{\mathrm{LL}}$ is the configuration tensor for a "fat tube" entanglement segment. This interpretation makes qualitative sense if it is understood that the "thin tube" is the tube which represents entanglements with all other chains, while the "fat tube" is the tube which represents entanglements with other long chains only (see [1,16,26,29]). In this context, we can define both the stretch along the "thin tube" $\lambda_{\text {thin }}=\left(\operatorname{Tr} \boldsymbol{A}_{\mathrm{L}} / 3\right)^{1 / 2}$ [as in Eq. (13)] and "fat tube" (i.e., stretch measured along the fat tube contour) $\lambda_{\text {fat }}=\left(\operatorname{Tr} \boldsymbol{A}_{\mathrm{LL}} / 3\right)^{1 / 2}$.

With regard to their underlying structure, the RDP and SRDP models are in total agreement to this point. However, the microscopic interpretation of the "off-diagonal" configuration $\boldsymbol{A}_{I J}$ (with $I \neq J$ ) tensors differs between the two models, and this leads to subtle differences in the constitutive formulations of the chain retraction and CCR terms.

\section{The RDP model}

The time evolution of the tensors $\boldsymbol{A}_{\mathrm{SS}}$ and $\boldsymbol{A}_{\mathrm{LL}}$ follow predictably the "classic" Rolie-Poly model presented in Sec. II A

$$
\begin{aligned}
\stackrel{\nabla}{\mathrm{ASS}}=- & \frac{\left(1+\beta_{\mathrm{th}}\right)}{\tau_{\mathrm{d}, \mathrm{S}}}\left(\boldsymbol{A}_{\mathrm{SS}}-\boldsymbol{I}\right)-\frac{2\left(1-\lambda_{\mathrm{S}}^{-1}\right)}{\tau_{\mathrm{s}, \mathrm{S}}} \mathrm{f}_{\mathrm{E}}\left(\lambda_{\mathrm{S}}\right) \\
& \times\left[\boldsymbol{A}_{\mathrm{SS}}+\beta_{\mathrm{CCR}} \lambda_{\mathrm{S}}^{2 \delta}\left(\boldsymbol{A}_{\mathrm{SS}}-\boldsymbol{I}\right)\right], \\
\stackrel{\nabla}{\mathrm{LL}}== & \frac{\left(1+\beta_{\mathrm{th}}\right)}{\tau_{\mathrm{d}, \mathrm{L}}}\left(\boldsymbol{A}_{\mathrm{LL}}-\boldsymbol{I}\right)-\frac{2\left(1-\lambda_{\mathrm{L}}^{-1}\right)}{\tau_{\mathrm{s}, \mathrm{L}}} \mathrm{f}_{\mathrm{E}}\left(\lambda_{\mathrm{L}}\right) \\
\times & {\left[\boldsymbol{A}_{\mathrm{LL}}+\beta_{\mathrm{CCR}} \lambda_{\mathrm{L}}^{2 \delta}\left(\boldsymbol{A}_{\mathrm{LL}}-\boldsymbol{I}\right)\right] . }
\end{aligned}
$$

Note that the rate of retraction of the long chains is proportional to $\left(\lambda_{\mathrm{L}}-1\right) \mathrm{f}_{\mathrm{E}}\left(\lambda_{\mathrm{L}}\right) / \tau_{\mathrm{s}, \mathrm{L}}$, and for short chains, it is proportional to $\left(\lambda_{\mathrm{S}}-1\right) \mathrm{f}_{\mathrm{E}}\left(\lambda_{\mathrm{S}}\right) / \tau_{\mathrm{S}, \mathrm{S}}$; this gives relaxation of stress from both the retraction itself and from the associated CCR events.

Now, we define the evolution equation of the "S/L" tensor representing the stress conformation tensor of the short chains arising from entanglements with the surrounding long chains

$$
\begin{aligned}
\stackrel{\nabla}{\boldsymbol{A}_{\mathrm{SL}}=} & -\frac{1}{\tau_{\mathrm{d}, \mathrm{S}}}\left(\boldsymbol{A}_{\mathrm{SL}}-\boldsymbol{I}\right)-\frac{2\left(1-\lambda_{\mathrm{S}}^{-1}\right)}{\tau_{\mathrm{s}, \mathrm{S}}} \mathrm{f}_{\mathrm{E}}\left(\lambda_{\mathrm{S}}\right) \boldsymbol{A}_{\mathrm{SL}} \\
& -\left(\boldsymbol{A}_{\mathrm{SL}}-\boldsymbol{I}\right)\left(\frac{\beta_{\mathrm{th}}}{\tau_{\mathrm{d}, \mathrm{L}}}+2 \beta_{\mathrm{CCR}} \frac{\left(1-\lambda_{\mathrm{L}}^{-1}\right)}{\tau_{\mathrm{s}, \mathrm{L}}} \mathrm{f}_{\mathrm{E}}\left(\lambda_{\mathrm{L}}\right) \lambda_{\mathrm{S}}^{2 \delta}\right) .
\end{aligned}
$$

The first and second terms on the RHS are, respectively, the short chain reptation and retraction contributions to the stress relaxation. The third term, in $\beta_{\text {th }}$, on the RHS allows the short chains to relax the stress associated with a L-chain entanglement whenever that entanglement is removed by CLFs or reptation. Again, we take $\beta_{\text {th }}=1$, which is the correct value to choose to be consistent with double reptation. The final term, in $\beta_{\mathrm{CCR}}$, on the RHS corresponds to the relaxation of short chain stress due to the CCR that occurs when a long chain retracts in flow and evacuates an entanglement with a neighboring short chain. Note that the rate of CCR is proportional to the rate at which long chains retract.

Similarly, we define the evolution equation for the stress conformation tensor of the long chains arising from entanglements with the surrounding short chains

$$
\begin{aligned}
{\stackrel{\nabla}{\boldsymbol{A}_{\mathrm{LS}}}=} & -\frac{1}{\tau_{\mathrm{d}, \mathrm{L}}}\left(\boldsymbol{A}_{\mathrm{LS}}-\boldsymbol{I}\right)-\frac{2\left(1-\lambda_{\mathrm{L}}^{-1}\right)}{\tau_{\mathrm{s}, \mathrm{L}}} \mathrm{f}_{\mathrm{E}}\left(\lambda_{\mathrm{L}}\right) \boldsymbol{A}_{\mathrm{LS}} \\
& -\left(\boldsymbol{A}_{\mathrm{LS}}-\boldsymbol{I}\right)\left(\frac{\beta_{\mathrm{th}}}{\tau_{\mathrm{d}, \mathrm{S}}}+2 \beta_{\mathrm{CCR}} \frac{\left(1-\lambda_{\mathrm{S}}^{-1}\right)}{\tau_{\mathrm{s}, \mathrm{S}}} \mathrm{f}_{\mathrm{E}}\left(\lambda_{\mathrm{S}}\right) \lambda_{\mathrm{L}}^{2 \delta}\right) .
\end{aligned}
$$

Clearly, the RDP model is asymmetric in its treatment of different chain species, i.e., $\boldsymbol{A}_{\mathrm{LS}} \neq \boldsymbol{A}_{\mathrm{SL}}$.

Equations (16)-(19) can be summarized as

$$
\stackrel{\nabla}{\boldsymbol{A}}_{i j}=-\boldsymbol{R}_{\mathrm{rep}, i j}-\boldsymbol{R}_{\mathrm{CR}, i j}-\boldsymbol{R}_{\mathrm{ret}, i j}-\boldsymbol{R}_{\mathrm{CCR}, i j},
$$

where $i$ and $j$ can be a long (L) or short (S) chain species and with the reptation (rep), CR, retraction (ret), and CCR relaxation terms being, respectively,

$$
\begin{gathered}
\boldsymbol{R}_{\mathrm{rep}, i j} \equiv \frac{1}{\tau_{\mathrm{d}, i}}\left(\boldsymbol{A}_{i j}-\boldsymbol{I}\right), \\
\boldsymbol{R}_{\mathrm{CR}, i j} \equiv \frac{\beta_{\mathrm{th}}}{\tau_{\mathrm{d}, j}}\left(\boldsymbol{A}_{i j}-\boldsymbol{I}\right), \\
\boldsymbol{R}_{\mathrm{ret}, i j} \equiv \frac{2\left(1-\lambda_{i}^{-1}\right)}{\tau_{\mathrm{s}, i}} \mathrm{f}_{\mathrm{E}}\left(\lambda_{i}\right) \boldsymbol{A}_{i j}, \\
\boldsymbol{R}_{\mathrm{CCR}, i j} \equiv 2 \beta_{\mathrm{CCR}} \frac{\left(1-\lambda_{j}^{-1}\right)}{\tau_{\mathrm{s}, j}} \mathrm{f}_{\mathrm{E}}\left(\lambda_{j}\right) \lambda_{i}^{2 \delta}\left(\boldsymbol{A}_{i j}-\boldsymbol{I}\right) .
\end{gathered}
$$

Together, Eqs. (12)-(15) and (20) define the RDP model.

\section{The SRDP model}

Although the RDP model can be shown to be derivable from the underlying tube model (see the supplementary material [51]), a functionally equivalent model (the SRDP model) can be developed by simply assuming that the microscopic picture of double reptation applies to all relevant nonlinear stress relaxation mechanisms.

The microscopic picture of double reptation is that of a temporary network with stress relaxation kinetics imported 
from monodisperse tube-based models. When a temporary cross-link (entanglement) is removed by $\mathrm{CR}$, either thermal or convective, the stress associated with that cross-link is relaxed entirely. Likewise, when one of the two chains relaxes its stretch (introducing "slack" into the entanglement), we may assume that a portion of that slack is taken on by the adjoining chain so that the two remain in mechanical equilibrium. In other words, the configuration of long chains at their point of entanglement with short chains, $\boldsymbol{A}_{\mathrm{LS}}$, always matches the configuration of short chains at their point of entanglement with long chains, $\boldsymbol{A}_{\mathrm{SL}}$. This is the microscopic interpretation for the "symmetric" aspect of the SRDP model.

To determine the rates of chain retraction and CCR, the SRDP model employs the $\lambda$-dependent kinetic coefficients from the monodisperse Rolie-Poly model without any further microscopic re-interpretation to account for polydispersity.

The resulting constitutive model is formulated as follows:

$$
\stackrel{\nabla}{\boldsymbol{A}_{i j}}=-\boldsymbol{R}_{\mathrm{rep}, i j}-\boldsymbol{R}_{\mathrm{CR}, i j}-\boldsymbol{R}_{\mathrm{ret}, i j}-\boldsymbol{R}_{\mathrm{CCR}, i j},
$$

where $i$ and $j$ can be a long (L) or short $(\mathrm{S})$ chain species and with the reptation (rep), retraction (ret), CR, and CCR terms being, respectively,

$$
\begin{gathered}
\boldsymbol{R}_{\mathrm{rep}, i j} \equiv \frac{1}{\tau_{\mathrm{d}, i}}\left(\boldsymbol{A}_{i j}-\boldsymbol{I}\right), \\
\boldsymbol{R}_{\mathrm{CR}, i j} \equiv \frac{\beta_{\mathrm{th}}}{\tau_{\mathrm{d}, j}}\left(\boldsymbol{A}_{i j}-\boldsymbol{I}\right), \\
\boldsymbol{R}_{\mathrm{ret}, i j} \equiv \frac{1}{2}\left[\frac{2\left(1-\lambda_{i}^{-1}\right)}{\tau_{\mathrm{s}, i}} \mathrm{f}_{\mathrm{E}}\left(\lambda_{i}\right)+\frac{2\left(1-\lambda_{j}^{-1}\right)}{\tau_{\mathrm{s}, j}} \mathrm{f}_{\mathrm{E}}\left(\lambda_{j}\right)\right] \boldsymbol{A}_{i j}, \\
\boldsymbol{R}_{\mathrm{CCR}, i j} \equiv \frac{1}{2} \beta_{\mathrm{CCR}}\left[\frac{2\left(1-\lambda_{i}^{-1}\right)}{\tau_{\mathrm{s}, i}} \mathrm{f}_{\mathrm{E}}\left(\lambda_{i}\right) \lambda_{i}^{2 \delta}+\frac{2\left(1-\lambda_{j}^{-1}\right)}{\tau_{\mathrm{s}, j}} \mathrm{f}_{\mathrm{E}}\left(\lambda_{j}\right) \lambda_{j}^{2 \delta}\right] \\
\times\left(\boldsymbol{A}_{i j}-\boldsymbol{I}\right) .
\end{gathered}
$$

Together, Eqs. (12)-(15) and (25) define the SRDP model. In contrast to the RDP model, here we find that $\boldsymbol{A}_{\mathrm{SL}}=\boldsymbol{A}_{\mathrm{LS}}$. For blends with many components $(N \gg 1)$, the SRDP model requires about half as many component configuration tensors $\left[N(N+1) / 2\right.$ vs $\left.N^{2}\right]$. Therefore, the SRDP model should run about twice as fast as the RDP model when considering CFD flow simulations of polydisperse melts.

Finally, in the spirit of the original Rolie-Poly model [10], we also provide a nonstretching version of the bidisperse SRDP model. As with the nonstretching Rolie-Poly model, the nonstretching SRDP model can be derived from the limit of asymptotically fast stretch relaxation dynamics in both chains:

$$
\boldsymbol{R}_{\mathrm{ret}, i j}=\frac{1}{2}\left(f_{i}+f_{j}\right) \boldsymbol{A}_{i j},
$$

$$
\begin{gathered}
\boldsymbol{R}_{\mathrm{CCR}, i j}=\frac{1}{2}\left(f_{i}+f_{j}\right) \beta_{\mathrm{CCR}}\left(\boldsymbol{A}_{i j}-\boldsymbol{I}\right), \\
f_{\mathrm{L}}=\frac{2}{3} \boldsymbol{\kappa}:\left(\boldsymbol{A}_{\mathrm{L}}+\phi_{\mathrm{S}}\left(\boldsymbol{A}_{\mathrm{L}}-\boldsymbol{A}_{\mathrm{S}}\right)\right), \\
f_{\mathrm{S}}=\frac{2}{3} \boldsymbol{\kappa}:\left(\boldsymbol{A}_{\mathrm{S}}+\phi_{\mathrm{L}}\left(\boldsymbol{A}_{\mathrm{S}}-\boldsymbol{A}_{\mathrm{L}}\right)\right),
\end{gathered}
$$

where $\boldsymbol{\kappa}$ is the velocity gradient tensor.

\section{Comparison of the two models in nonlinear shear flow}

The difference between the RDP and SRDP models are briefly summarized in Table I.

In more detail, comparing the RDP and SRDP model formulations, we see that the two primarily differ by the way that chain retraction and CCR relax the stress associated with entanglements between long and short chains. Considering only the effect of long chain retraction (and corresponding CCR) on entanglements of long/short chains, the RDP model predicts that stress on the long chain side is relaxed only by retraction and stress on the short chain side is relaxed only by CCR. By contrast, the SRDP model predicts that the stress relaxation by retraction and CCR are evenly distributed between the two chains.

However, when $\beta_{\mathrm{CCR}}=1$, the rate of CCR is necessarily equal to the rate of retraction, and so the overall relaxation of stress is roughly the same irrespective of how retraction and CCR are divided between the two chains. Similar arguments can be made for the effects of short chain retraction (and corresponding CCR). This physical argument for agreement of the two models is predicated on $\beta_{\mathrm{CCR}}$ equal to unity, and unsurprisingly, we find that for $\beta_{\mathrm{CCR}}=0$, disagreements between the two models are more evident, especially in the individual component tensors (e.g., $\boldsymbol{A}_{\mathrm{LS}}$ vs $\boldsymbol{A}_{\mathrm{SL}}$ ). Fortunately, even in this case the total stresses still seem to agree for a wide range of shear rates: If the SRDP model (relative to RDP) underestimates the stress in long chains and overestimates the stress in short chains, then to leading order the total stress is apparently unaffected!

A more detailed quantitative comparison of the two models is provided in the supplementary material [51] for the interested reader. Overall, we find that the RDP and SRDP models are in quantitative agreement to within $\sim 10 \%$ for $|\boldsymbol{\kappa}| \tau_{\mathrm{s}, \mathrm{L}}<1$. Quantitative disagreement between the two models is most evident when short chains are stretching but

TABLE I. Brief summary of the differences between the RDP and SRDP models.

\begin{tabular}{lcc}
\hline \hline Effect & RDP & SRDP \\
\hline Orientation and CR relaxation of $i j$ & Symmetric & Symmetric \\
Stretch relaxation of $i j$ from retraction of & $\begin{array}{c}i \text { chain, but } \\
\text { not } j \text { chain }\end{array}$ & $\begin{array}{c}\text { Both } i \text { and } j \\
\text { chain }\end{array}$ \\
CCR relaxation of $i j$ from retraction of & $\begin{array}{c}j \text { chain, but } \\
\text { not } i \text { chain }\end{array}$ & $\begin{array}{c}\text { Both } i \text { and } j \\
\text { chain }\end{array}$ \\
\hline \hline
\end{tabular}


not near full extension, $|\boldsymbol{\kappa}| \tau_{\mathrm{s}, \mathrm{S}} \sim \mathcal{O}(1)$. However, even under these conditions, qualitative agreement is still quite good and neither model shows any clear advantage over the other when compared against the available experimental data.

\section{Enhanced stretch relaxation time}

The onset of chain stretch and emergent extension hardening in the nonlinear rheological response of molten binary blends (of long and short polymer chains) is set by an effective stretch relaxation time of the long chains. It has been found experimentally [30] that, for some particular blends in region $3 \mathrm{c}$ of the modified Viovy diagram, Fig. 1(b), this effective stretch relaxation time of the long chains initially increases proportionally to the inverse volume fraction of long chains $\phi_{\mathrm{L}}^{-\alpha}$, where $\alpha$ is the dilution exponent for entanglements (we assume $\alpha=1$, which is the correct exponent if one assumes entanglements are binary events; it is also the value favored by recent experimental evidence, e.g., [34]). In elongation experiments, if the flow rate exceeds the inverse of the stretch relaxation time of the long molecules, they are stretched by the flow, i.e., their length along the tube contour grows. The macroscopic consequence of chain stretch is elongation hardening, i.e., the elongational viscosity, $\eta_{\mathrm{E}}^{+}$, grows above the linear viscoelastic envelope (LVE) prediction.

A detailed theoretical framework for a bidisperse blend of entangled polymers has been established by Read et al. [26], where the enhanced stretch relaxation time appears naturally within the theory: This occurs for blend parameters which (as noted above) fall within region $3 \mathrm{c}$ of the modified Viovy diagram. In terms of the short chain length, they should be sufficiently short that the long chains can locally explore the fat tube by CR motion; on the other hand, the short chains should not be too short, otherwise, rapid CR along the fat tube accelerates the long chain stretch relaxation: for more details, see [26,30].

Although much simpler, the RDP and SRDP models contain the same enhanced stretch relaxation time arising for the same physical reasons as [26]. We consider the limit in which the short chain dynamics are fast compared to the stretch relaxation of the long chains $\left(\tau_{\mathrm{d}, \mathrm{S}} / \tau_{\mathrm{s}, \mathrm{L}} \ll 1\right)$ and also fast compared to flow time scales $\left(\dot{\varepsilon} \tau_{\mathrm{d}, \mathrm{S}} \ll 1\right.$, where $\dot{\varepsilon}$ is the typical strain rate). In this case, any tensors related to the short chains will relax quickly on the important time scales of either long-chain stretch relaxation, or of flow, and in particular, we have $\boldsymbol{A}_{\mathrm{LS}} \approx \boldsymbol{I}$. Hence the stretch, $\lambda_{\mathrm{L}}$, of the L-chain, defined in Eq. (13), is

$$
\begin{aligned}
\lambda_{\mathrm{L}}^{2}= & \frac{1}{3}\left(\phi_{\mathrm{S}} \operatorname{Tr} \boldsymbol{A}_{\mathrm{LS}}+\phi_{\mathrm{L}} \operatorname{Tr} \boldsymbol{A}_{\mathrm{LL}}\right) \\
& \approx \phi_{\mathrm{S}}+\frac{1}{3} \phi_{\mathrm{L}} \operatorname{Tr} \boldsymbol{A}_{\mathrm{LL}}, \quad \tau_{\mathrm{d}, \mathrm{S}} / \tau_{\mathrm{s}, \mathrm{L}} \ll 1 .
\end{aligned}
$$

We take the time derivative of Eq. (34), and use Eq. (17) keeping only the stretch relaxation term, to obtain

$$
2 \lambda_{\mathrm{L}} \frac{\mathrm{d} \lambda_{\mathrm{L}}}{\mathrm{d} t} \approx \frac{\phi_{\mathrm{L}}}{3}\left(-\frac{2}{\tau_{\mathrm{s}, \mathrm{L}}}\left(1-\lambda_{\mathrm{L}}^{-1}\right) \operatorname{Tr} \boldsymbol{A}_{\mathrm{LL}}\right) .
$$

We then use Eq. (34) to arrive at

$$
\frac{\mathrm{d} \lambda_{\mathrm{L}}}{\mathrm{d} t}=-\frac{1}{\tau_{\mathrm{s}, \mathrm{L}}} \frac{\left(1-\lambda_{\mathrm{L}}^{-1}\right)\left(\lambda_{\mathrm{L}}^{2}-\phi_{\mathrm{S}}\right)}{\lambda_{\mathrm{L}}} .
$$

We write the stretch as $\lambda_{\mathrm{L}}=1+\ell_{\mathrm{L}}$, where $\ell_{\mathrm{L}}$ is the "extra" stretch. Therefore, we have

$$
1-\lambda_{\mathrm{L}}^{-1}=\ell_{\mathrm{L}} /\left(1+\ell_{\mathrm{L}}\right), \quad \lambda_{\mathrm{L}}^{2}-\phi_{\mathrm{S}}=\phi_{\mathrm{L}}+2 \ell_{\mathrm{L}}+\ell_{\mathrm{L}}^{2},
$$

so Eq. (36) gives the time evolution of the extra stretch

$$
\begin{aligned}
\frac{\mathrm{d} \ell_{\mathrm{L}}}{\mathrm{d} t}= & -\frac{1}{\tau_{\mathrm{s}, \mathrm{L}}} \frac{\ell_{\mathrm{L}}\left(\phi_{\mathrm{L}}+2 \ell_{\mathrm{L}}+\ell_{\mathrm{L}}^{2}\right)}{\left(1+\ell_{\mathrm{L}}\right)^{2}} \\
& \approx\left\{\begin{array}{ll}
-\frac{\phi_{\mathrm{L}}}{\tau_{\mathrm{s}, \mathrm{L}}} \ell_{\mathrm{L}}, & \ell_{\mathrm{L}} \ll 1 \\
-\frac{1}{\tau_{\mathrm{s}, \mathrm{L}}} \ell_{\mathrm{L}}, & \ell_{\mathrm{L}} \gg 1
\end{array} .\right.
\end{aligned}
$$

We see that the "effective" stretch relaxation time of the L-chains at small stretch $\left(\ell_{\mathrm{L}} \ll 1\right)$ is

$$
\tau_{\mathrm{s}, \mathrm{L}}^{\mathrm{eff}}=\frac{\tau_{\mathrm{s}, \mathrm{L}}}{\phi_{\mathrm{L}}}
$$

The stretch relaxation time is, therefore, renormalized by a factor $\phi_{\mathrm{L}}^{-1}>1$, so, the long chains (blended with short chains) will start to show the stretch effects at smaller flow rates than if there were no short chains. In uniaxial elongation, we therefore expect hardening for flow rates such that $\dot{\varepsilon} \tau_{\mathrm{s}, \mathrm{L}}^{\text {eff }}>1$. At large stretch, $\ell_{\mathrm{L}} \gg 1$, there is no renormalization of the stretch relaxation time.

We illustrate this phenomenon in Fig. 2, where the predictions of our RDP model in uniaxial extension are shown,

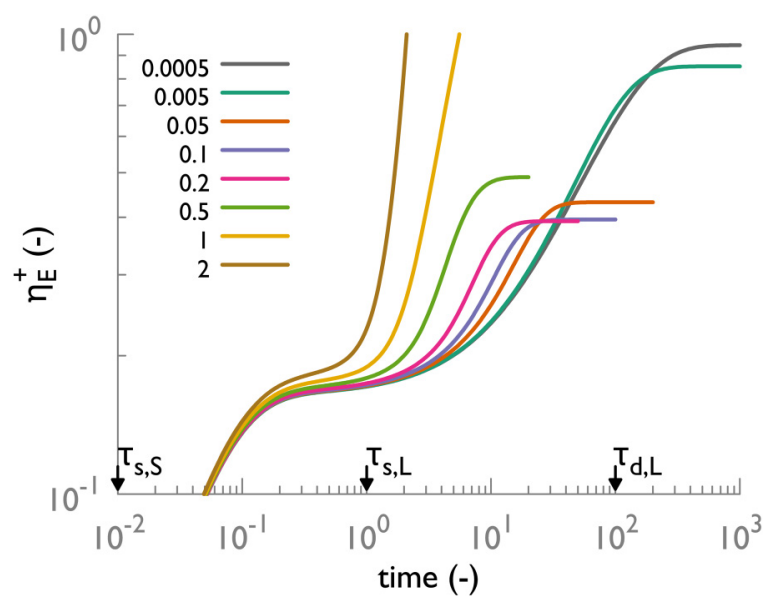

FIG. 2. Illustration of the enhanced stretch relaxation time using the RDP model. Logarithms of the stress growth coefficient in elongation, $\eta_{\mathrm{E}}^{+}$as a function of time for different elongation rates indicated in the figure legend for a blend of S-chains $\left(\tau_{\mathrm{d}, \mathrm{S}}=0.05, \tau_{\mathrm{s}, \mathrm{S}}=0.01\right)$ with, $\phi_{\mathrm{L}}=0.05$, L-chains $\left(\tau_{\mathrm{d}, \mathrm{L}}=100, \tau_{\mathrm{s}, \mathrm{L}}=1\right)$, in arbitrary units. We set $\lambda_{\max } \gg 1$. According to Eq. (39), the enhanced stretch relaxation time is $\tau_{\mathrm{s}, \mathrm{L}} / \phi_{\mathrm{L}}=20$. Time scales are placed on axis to aid interpretation: $\tau_{\mathrm{d}, \mathrm{L}}$ indicates the terminal relaxation time of the LVE, and short chains are not stretched as all flow rates are below $\tau_{\mathrm{s}, \mathrm{S}}^{-1}$. Although elongation hardening is expected at rates above $\tau_{\mathrm{s}, \mathrm{L}}^{-1}$, the enhanced stretch relaxation time can be seen to give hardening at much lower rates than this. 

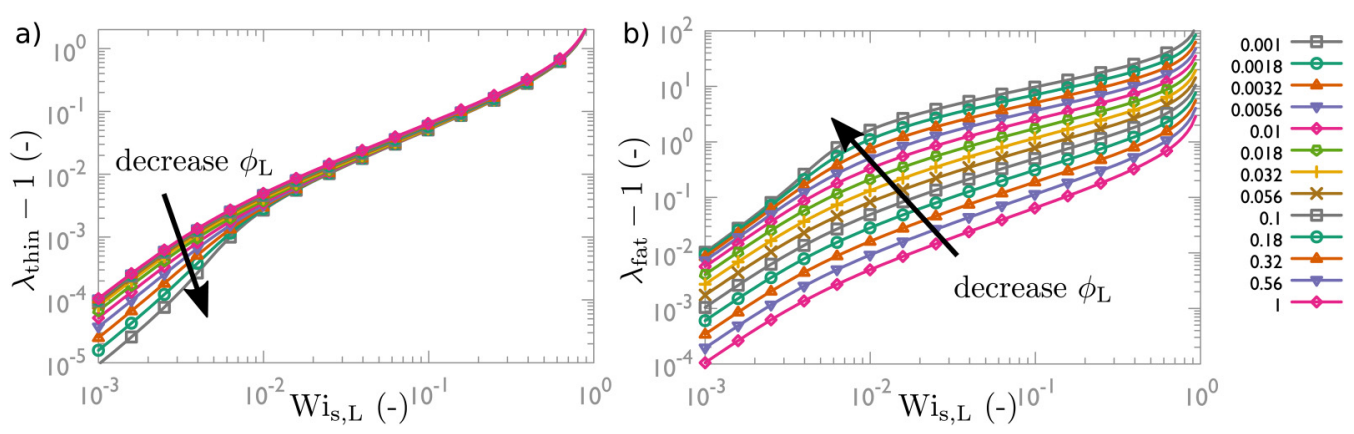

FIG. 3. Effect of increasing dilution on the steady state stretch in (a) the thin tube, $\lambda_{\text {thin }}=\left(\operatorname{Tr} \boldsymbol{A}_{\mathrm{L}} / 3\right)^{1 / 2}$ and (b) the fat tube, $\lambda_{\text {fat }}=\left(\operatorname{Tr} \boldsymbol{A}_{\mathrm{LL}} / 3\right)^{1 / 2}$, as a function of $\mathrm{Wi}_{\mathrm{s}, \mathrm{L}} \equiv \dot{\varepsilon} \tau_{\mathrm{s}, \mathrm{L}}$. The volume fraction of long chains, $\phi_{\mathrm{L}}$, is indicated in the figure legend. Relaxation time parameters are the same as in Fig. 2. Note that stretches diverge for $\mathrm{Wi}_{\mathrm{s}, \mathrm{L}}>1$ since finite extensibility is not included here.

in arbitrary units, effectively without finite extensibility $\left(\lambda_{\max } \gg 1\right)$. Short chains $\left(\tau_{\mathrm{d}, \mathrm{S}}=0.05, \tau_{\mathrm{s}, \mathrm{S}}=0.01\right)$ are blended with $5 \%$ of long chains $\left(\tau_{\mathrm{d}, \mathrm{L}}=100, \tau_{\mathrm{s}, \mathrm{L}}=1\right)$. When the elongation rate, $\dot{\varepsilon}$, is small, the stress growth coefficient (viscosity), $\eta_{\mathrm{E}}^{+}$, follows the LVE. Note that $\eta_{\mathrm{E}}^{+} \equiv\left(\sigma_{x x}-\sigma_{y y}\right) / \dot{\varepsilon}$ in uniaxial elongation along the $x$-axis. The onset of elongation hardening is clearly seen for $\dot{\varepsilon}=0.05$ and above. This is in agreement with Eq. (39) as $\tau_{\mathrm{s}, \mathrm{L}}=1$ and $\phi_{\mathrm{L}}=0.05$ so that $\tau_{\mathrm{s}, \mathrm{L}}^{\text {eff }}=20$, and elongation hardening is expected for $\dot{\varepsilon} \tau_{\mathrm{s}, \mathrm{L}}^{\text {eff }}>1$. Another feature of Fig. 2 is that the stress grows but then reaches a maximum for flow rates between 0.05 and 1 ; but then it continues to grow for larger flow rates. This has to do with nonlinear terms in Eq. (38) since, for large $\ell_{\mathrm{L}}$, the effective relaxation time returns to simply $\tau_{\mathrm{s}, \mathrm{L}}^{-1}$. Hence, for $\left(\tau_{\mathrm{s}, \mathrm{L}}^{\text {eff }}\right)^{-1}<\dot{\varepsilon}<\left(\tau_{\mathrm{s}, \mathrm{L}}\right)^{-1}$, we expect the onset of extension hardening, but for it to be limited when nonlinearities become important.

We now illustrate this further by examining the stretch in thin and fat tubes in detail. As noted earlier in Sec. II B, the tensor $\boldsymbol{A}_{\mathrm{L}}$ may be considered to be the configuration tensor for a "thin tube" entanglement segment, with associated thin tube stretch $\lambda_{\text {thin }}=\left(\operatorname{Tr} \boldsymbol{A}_{\mathrm{L}} / 3\right)^{1 / 2}$, while $\boldsymbol{A}_{\mathrm{LL}}$ is the configuration tensor for a "fat tube" entanglement segment, with fat tube stretch $\lambda_{\text {fat }}=\left(\operatorname{Tr} \boldsymbol{A}_{\mathrm{LL}} / 3\right)^{1 / 2}$. In Fig. 3, we present the steady state values of $\lambda_{\text {thin }}$ (a), and $\lambda_{\text {fat }}$ (b) as a function of $\mathrm{Wi}_{\mathrm{s}, \mathrm{L}} \equiv \dot{\varepsilon} \tau_{\mathrm{s}, \mathrm{L}}$, for different dilutions, $\phi_{\mathrm{L}}$. Note that the thin tube diameter is fixed for all dilutions, but the fat tube diameter gets wider with increasing dilution (i.e., with decreasing $\phi_{\mathrm{L}}$ ).

For interesting blends (e.g., short chains with long chains), $\mathrm{CR}$ from the short chains is fast, so the thin tube locally equilibrates in the fat tube. When we consider the stress, it is natural to look at the orientation and stretch at the coarsegrained lengthscale of the fat tube. When there is significant stretch at the fat tube lengthscale, we see extension hardening.

From Fig. 3, we note the following:

(i) For intermediate and large values of $\mathrm{Wi}_{\mathrm{s}, \mathrm{L}}$, the steady state stretch in the thin tube is essentially unaffected by increasing dilution, i.e., as $\phi_{\mathrm{L}}$ decreases. The only differences seen are for the extremely small stretches at rates where $\dot{\varepsilon} \tau_{\mathrm{d}, \mathrm{L}}<1$, i.e., where the stretch is additionally relaxed by fat tube reorientation.

(ii) The steady state stretch in the fat tube increases with increasing dilution, i.e., as $\phi_{\mathrm{L}}$ decreases, and the lowest flow rate at which significant stretch in the fat tube is observed gets smaller with increasing dilution. In this sense, the effective stretch relaxation time along the fat tube increases as the long chains are diluted. Consequently, since stress is obtained from orientation and stretch at the fat tube lengthscale, this means we observe extension hardening at lower flow rates as long chains are diluted by shorter ones.

\section{Comparison with experimental data}

In this section, we pursue a quantitative comparison of the RDP model predictions against experimental data for the four bidisperse melts of [26]. As we have noted previously, the predictions from the SRDP model are indistinguishable from those of RDP under the flow conditions represented by this data. The majority of the parameters in the model are fixed by chemistry, taken from the literature values, or calculated therefrom. For qualitative purposes, the RDP model requires no further (free) parameters: The model correctly predicts the overall trends in the data and could be used (for example) to make qualitative predictions in complex flow fields. For quantitative interests (i.e., closely matching the experimental data), we allow a single fitting parameter to correct for departures from $\alpha=1$ entanglement probabilities (predicted by double reptation and the chemical composition of the blend), as discussed in more detail below. This fitting parameter is set by the linear rheology alone so that predictions of the nonlinear rheology (shear and extension) are made without any further intervention. The primary effect of this additional parameter is to change the orientation relaxation time and modulus associated with the long chains in the blends, so it makes no qualitative change to the structure of the model.

TABLE II. Parameters of four binary blends from [26] and from [30] and [35] for the last two columns.

\begin{tabular}{lcccc}
\hline \hline & PI226_23_20 & PI226_23_40 & PI483_34_40 & PS blend 2 \\
\hline$T\left({ }^{\circ} \mathrm{C}\right)$ & 25 & 25 & 25 & 130 \\
$\phi_{\mathrm{L}}$ & 0.2 & 0.4 & 0.4 & 0.1437 \\
$G_{\mathrm{e}}(\mathrm{kPa})$ & 595.5 & 595.5 & 595.5 & 276 \\
$M_{\mathrm{L}}(\mathrm{kg} / \mathrm{mol})$ & 226 & 226 & 483.1 & 390 \\
$M_{\mathrm{S}}(\mathrm{kg} / \mathrm{mol})$ & 23.4 & 23.4 & 33.6 & 51.7 \\
$M_{\mathrm{e}}(\mathrm{kg} / \mathrm{mol})$ & 4.816 & 4.816 & 4.816 & 18.1 \\
$\tau_{\mathrm{e}}(\mathrm{s})$ & $1.314 \times 10^{-5}$ & $1.314 \times 10^{-5}$ & $1.314 \times 10^{-5}$ & 0.91 \\
\hline \hline
\end{tabular}



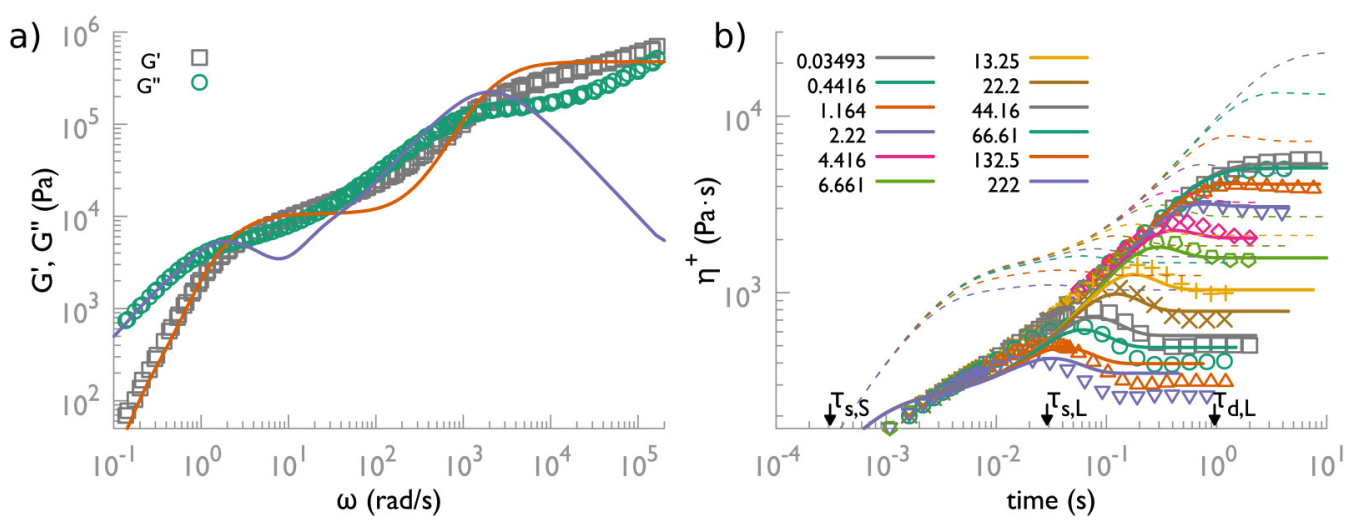

FIG. 4. PI226_23_20. Experimental data (symbols) and model predictions using $\tilde{\phi}_{\mathrm{L}}$ (solid and dashed lines). (a) LVE. (b) Nonlinear rheology, shear stress growth coefficients $\left(\dot{\gamma}=\{0.03493,0.4416,1.164,2.22,4.416,6.661,13.25,22.2,44.16,66.61,132.5,222\} \mathrm{s}^{-1}\right)$. Thin dashed lines are predictions using Eq. (41) for $\tau_{\mathrm{d}}$.

\section{Fixed parameters}

We use the parameters $\left\{\tau_{\mathrm{e}}, M_{\mathrm{e}}, M_{\mathrm{L}}, M_{\mathrm{S}}, G_{\mathrm{e}}\right\}$, provided in Table II of [26], for four blends of (long and short) linear monodisperse entangled chains $[26,30,35]$. We reproduce these parameters in Table II. For these parameters, and the parameters derived from them below, we use the "G-definition" from the options described by [36].

\section{Calculated parameters}

a. Stretch relaxation times. The stretch relaxation times of the short (S) and long (L) chains are computed from these fixed parameters, Table II, as follows:

$$
\tau_{\mathrm{s}, \mathrm{S}}=Z_{\mathrm{S}}^{2} \tau_{\mathrm{e}}, \quad \tau_{\mathrm{s}, \mathrm{L}}=Z_{\mathrm{L}}^{2} \tau_{\mathrm{e}}
$$

where $Z=M / M_{\mathrm{e}}$ is the entanglement number, $M$ is the molecular mass of the S- or L-chains, $M_{\mathrm{e}}$ is the entanglement molecular mass, and $\tau_{\mathrm{e}}$ is the Rouse time of an entanglement segment.

b. CLF correction to reptation times. For monodisperse polymer melts of asymptotically large entanglement number $(Z \gg 1)$, the average reptation time is given by

$$
\tau_{\mathrm{d}}=3 Z^{3} \tau_{\mathrm{e}}
$$

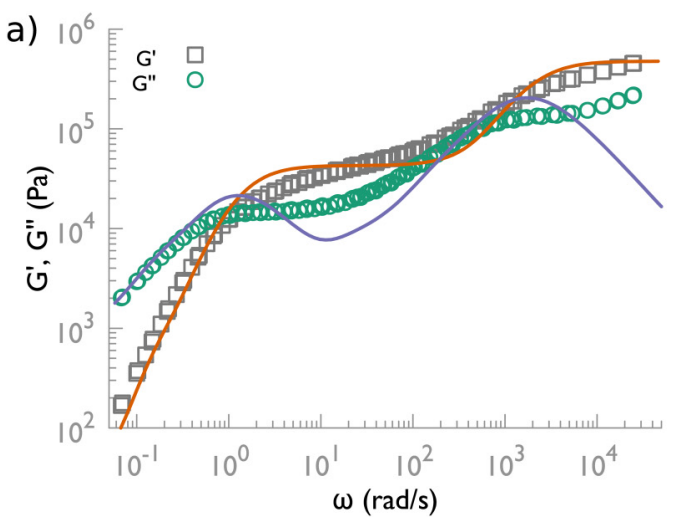

Using Eq. (41) for each reptation time in our model gives the thin dashed lines in Figs. 4-7, which offers a good qualitative description of the data. We can improve the model prediction by noting that, for chains with finite $Z$, fluctuations in the polymer's contour length (CLF) can facilitate a quantitatively significant speed-up of stress relaxation dynamics. In this case, we can incorporate the effects of CLF as a correction to the "bare" reptation time, as discussed in [37,38], such that the relaxation time $\tau_{\mathrm{d}}$ becomes the reptation time shortened by CLF. More precisely, we define $\tau_{\mathrm{d}}$ by

$$
\begin{aligned}
& \tau_{\mathrm{d}}=3 Z^{3} \tau_{\mathrm{e}} \mathrm{f}_{\mu}(Z), \quad \text { with } \\
& \mathrm{f}_{\mu}(Z)=1-\frac{2 C_{1}}{Z^{1 / 2}}+\frac{C_{2}}{Z}+\frac{C_{3}}{Z^{3 / 2}},
\end{aligned}
$$

where the function $\mathrm{f}_{\mu}(Z)$ accounts for the CLF effects. The coefficients are $C_{1}=1.69, C_{2}=4.17$, and $C_{3}=-1.55$ [37].

Taking a similar approach for our binary blend, we define

$$
\tau_{\mathrm{d}, \mathrm{S}}=3 Z_{\mathrm{S}}{ }^{3} \tau_{\mathrm{e}} \mathrm{f}_{\mu}\left(Z_{\mathrm{S}}\right), \quad \tau_{\mathrm{d}, \mathrm{L}}=3 Z_{\mathrm{L}}{ }^{3} \tau_{\mathrm{e}} \mathrm{f}_{\mu}\left(\tilde{Z}_{\mathrm{L}}\right),
$$

where $\tilde{Z}_{\mathrm{L}}=\phi_{\mathrm{L}} Z_{\mathrm{L}}$ is the effective number of entanglements along the long chain, after accounting for the CR effects (dilution from the short chains). The expression for $\tau_{\mathrm{d}, \mathrm{L}}$ is as given by Read et al. [16] for blends in region 3c of the Viovy

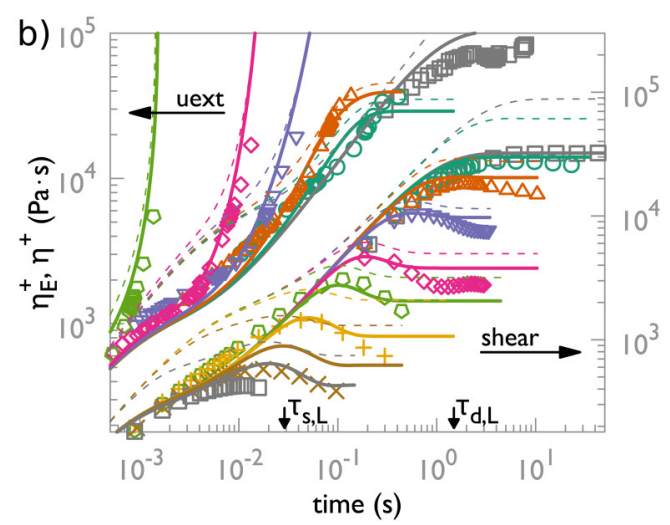

FIG. 5. PI226_23_40. Experimental data (symbols) and model predictions using $\tilde{\phi}_{\mathrm{L}}$ (solid and dashed lines). (a) LVE. (b) Nonlinear rheology, stress growth coefficients. Elongation $\left(\dot{\varepsilon}=\{0.2321,6.796,22.65,67.64,225.5\} \mathrm{s}^{-1}\right)$; shear $\left(\dot{\gamma}=\{0.02903,0.2903,0.9676,2.903,9.676,22.65,67.96,226.5,679.6\} \mathrm{s}^{-1}\right)$, top and bottom curves, respectively. Thin dashed lines are predictions using Eq. (41) for $\tau_{\mathrm{d}}$. 

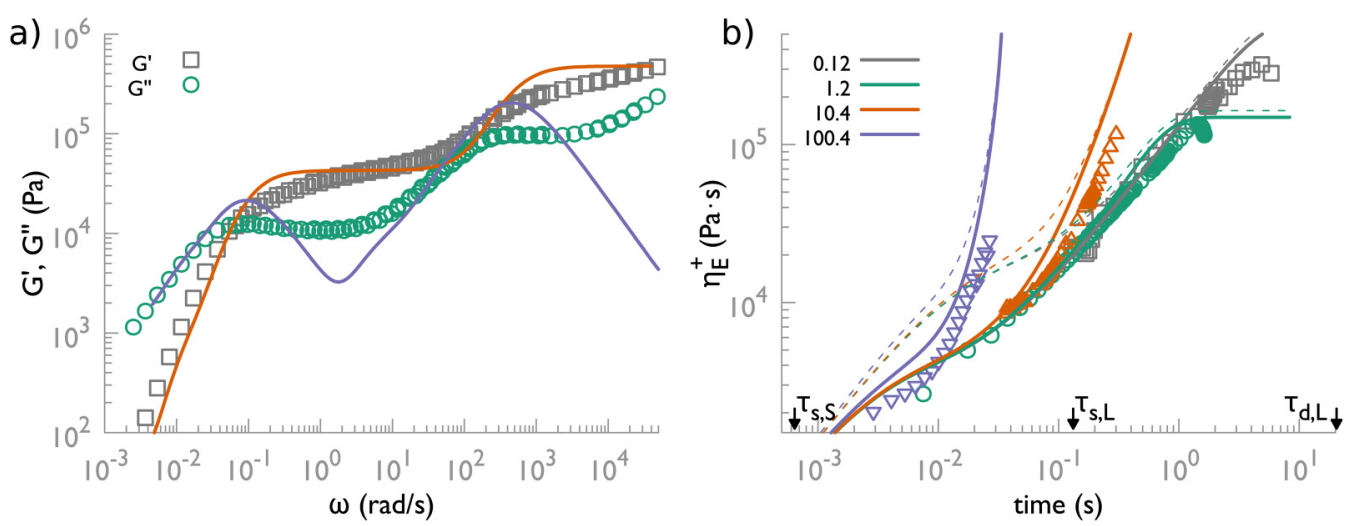

FIG. 6. PI483_34_40. Experimental data (symbols) and model predictions using $\tilde{\phi}_{\mathrm{L}}$ (solid and dashed lines). (a) LVE. (b) Nonlinear rheology, stress growth coefficients in elongation $\left(\dot{\varepsilon}=\{0.12,1.2,10.4,100.4\} \mathrm{s}^{-1}\right)$. Thin dashed lines are predictions using Eq. (41) for $\tau_{\mathrm{d}}$.

diagram [see Fig. 1(b)] and corresponds to the case where chains transport is fastest along the thin tube (hence the use of bare reptation time $3 Z_{\mathrm{L}}{ }^{3} \tau_{\mathrm{e}}$ ) but where there is freedom for CLF along the fat tube [hence the diluted value of $\tilde{Z}_{\mathrm{L}}$ is used in the CLF correction $\mathrm{f}_{\mu}\left(\tilde{Z}_{\mathrm{L}}\right)$ ]. Read et al. [16] also provide expressions that can be used in other regions of the Viovy diagram. As the short chains become longer and CR is slowed down, CLF in the fully diluted fat tube is no longer possible [and ultimately, CLF is only possible in the thin tube so that Eq. (42) is recovered]. For faster CR (shorter short chains) blends move into region 4 of the Viovy diagram, and are further accelerated by CR-induced reptation along the fat tube.

c. Plateau modulus. Doi-Edwards theory predicts the plateau modulus to be $4 / 5$ of the rubber modulus, $G_{\mathrm{e}}=\rho R T / M_{\mathrm{e}}$, because entanglements do allow longitudinal motion along the tube and therefore are different from cross-links [6]. We use the "G-definition" $[36,39,40]$ of the plateau modulus, which includes this $4 / 5$ prefactor from Doi-Edwards theory. Hence, the value of the (experimentally observed) plateau modulus, $G_{N}^{0}$, that we use in Eq. (12), is taken as

$$
G_{N}^{0}=\frac{4 \rho R T}{5} \frac{4}{M_{\mathrm{e}}}=\frac{4}{5} G_{\mathrm{e}}
$$

where $G_{\mathrm{e}}$ is the value reported in Table II.
In Table III, we summarize the calculated parameters used to produce the predictions in Figs. 4-7.

\section{Fitting parameter $\tilde{\phi}_{\mathrm{L}}$}

The RDP model requires no free parameters for qualitative purposes, however, for quantitative comparisons, we allow a single fitting parameter to correct for departures from the ideal binary picture of entanglement probabilities $(\alpha=1)$. The double reptation approximation assumes that the number of entanglements between chains $i$ and $j$ scales as $\phi_{i} \phi_{j}$, and so in a sense the RDP and SRDP models treat volume fractions as a proxy for computing entanglement densities. Experimentally, however, the long chains in a bidisperse melt are often found to be less self-entangled than a simple double reptation approximation would predict; this effect is especially visible when the relaxation times of short and long chains are well separated. For such melts, the double reptation approximation predicts that the effective entanglement molecular weight in the long chains should scale with $1 / \phi_{\mathrm{L}}$. However, this assumes that all parts of the long chains provide "slow" entanglement constraints on the time scale of the terminal relaxation. In reality, CLFs and the faster reptation modes relax the outer portions of long chains relatively quickly so that by the time of the terminal relaxation the effective dilution of the entanglements constraining the long chains is reduced to a smaller fraction $\tilde{\phi}_{\mathrm{L}} \leq \phi_{\mathrm{L}}$. Such an
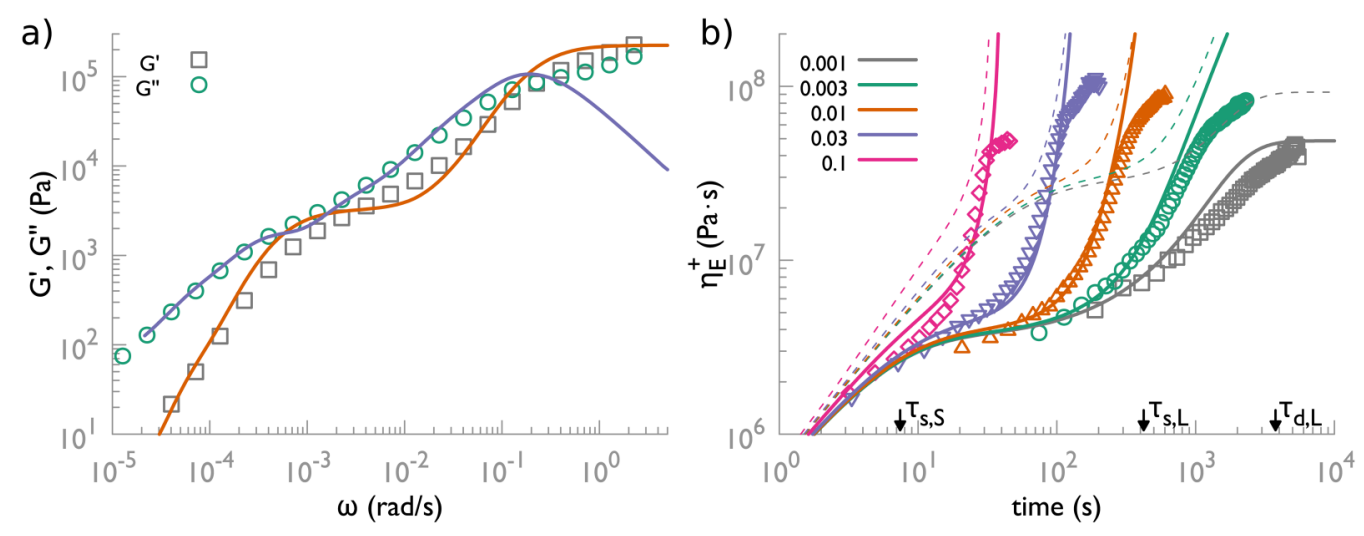

FIG. 7. PS Blend 2. Experimental data (symbols) and model predictions using $\tilde{\phi}_{\mathrm{L}}$ (solid and dashed lines). (a) LVE. (b) Nonlinear rheology, stress growth coefficients in elongation $\left(\dot{\varepsilon}=\{0.001,0.003,0.01,0.03,0.1\} \mathrm{s}^{-1}\right)$. Thin dashed lines are predictions using Eq. (41) for $\tau_{\mathrm{d}}$. 
TABLE III. Computed parameters using Eqs. (40) and (43). $\phi_{\mathrm{L}}$ is adjusted to $\tilde{\phi}_{\mathrm{L}}$ for best fit in the linear rheology. The percentage change is shown in brackets.

\begin{tabular}{lcccc}
\hline \hline & PI226_23_20 & PI226_23_40 & PI483_34_40 & PS blend 2 \\
\hline$G_{N}^{0}(\mathrm{kPa})$ & 476 & 476 & 476 & 221 \\
$\tau_{\mathrm{d}, \mathrm{L}}(\mathrm{s})$ & 0.959 & 1.49 & 20.4 & 3860 \\
$\tau_{\mathrm{s}, \mathrm{L}}(\mathrm{s})$ & $2.89 \times 10^{-2}$ & $2.89 \times 10^{-2}$ & 0.132 & 422 \\
$\tau_{\mathrm{d}, \mathrm{S}}(\mathrm{s})$ & $8.14 \times 10^{-4}$ & $8.14 \times 10^{-4}$ & $3.13 \times 10^{-3}$ & 8.84 \\
$\tau_{\mathrm{s}, \mathrm{S}}(\mathrm{s})$ & $3.10 \times 10^{-4}$ & $3.10 \times 10^{-4}$ & $6.40 \times 10^{-4}$ & 7.42 \\
$\tilde{\phi}_{\mathrm{L}}$ & $0.15(-25 \%)$ & $0.3(-25 \%)$ & $0.3(-25 \%)$ & $0.12(-17 \%)$ \\
\hline \hline
\end{tabular}

effect was noted by Shahid et al. [34] in examining dilution by short, oligomeric chains: Despite the actual dilution exponent being equal to one, the apparent dilution exponent for terminal modulus was found to be greater than one, because of the change in the relaxation spectrum toward a greater proportion of fast modes as the number of long chain entanglements decreased. Likewise, Read et al. [16] noted a similar effect in their assessment [based on Eq. (43)] of the change in terminal time for long chains upon dilution. Hence, without further correction our model will tend to overestimate the stress contribution from long chains.

It is beyond the scope of the present report to account for this effect in detail. Nonetheless, as a practical (albeit phenomenological) solution, we can simply replace the true volume fraction of long chains with its diluted value, $\phi_{\mathrm{L}}=\tilde{\phi}_{\mathrm{L}}$ and $\phi_{\mathrm{S}}=1-\tilde{\phi}_{\mathrm{L}}$, where the diluted fraction $\tilde{\phi}_{\mathrm{L}}$ is a free (fitting) parameter assumed to take a value a little less than the total long chain fraction. The value of $\tilde{\phi}_{\mathrm{L}}$ is determined from a best fit between the model and the experimental data of the linear rheology, cf. Figs. 4(a)-7(a), giving the values reported in Table III. A similar fitting parameter was used in [26]. Note that with only two relaxation times, we are able to obtain a reasonably good match (but not perfect) between the linear rheology predictions and the data. Other than the tube model parameters listed in Table II, $\tilde{\phi}_{\mathrm{L}}$ is the only fitting parameter used in this section. Subsequently, the nonlinear rheology predictions are produced without extra fitting parameters.

\section{Nonlinear rheology predictions}

Nonlinear data and model predictions for the viscosity [in shear $\eta^{+} \equiv \sigma_{x y} / \dot{\gamma}$ and in uniaxial elongation
$\left.\eta_{\mathrm{E}}^{+} \equiv\left(\sigma_{x x}-\sigma_{y y}\right) / \dot{\varepsilon}\right]$ are shown in Figs. 4(b)-7(b) using Eqs. (41) (dashed lines) and (43) (lines) for the reptation times. In the former case, the RDP model captures the qualitative features of the experimental data, while in the latter case, the RDP model quantitatively matches the experimental data in elongation at all elongation rates. Since an enhanced stretch time is evident in the data (see, e.g., [30]), we conclude that our model likewise provides a physically realistic description of the same phenomena. However, in shear, the weaknesses of our small-mode constitutive model are more apparent. Whereas the detailed model of [26] quantitatively matched the same data over the entire range of shear rate, the RDP model tends to overpredict shear stresses at the highest shear rates considered in the experimental data presented here. Here, we interpret the success of the detailed model of Read (and the failure of RDP) in terms of shear thinning physics that emerge only in a full chain constitutive model. Specifically, stretch relaxation in the full chain model pulls the outer chain sections into the more oriented tube segments surrounding the chain center; this is an additional alignment mechanism which produces greater shear thinning. Note also that other authors [41-44] have found it necessary to include an orientation dependent friction coefficient in fast elongational flow, which could also be used here to better model the transition to steady state elongational flow. The only data here which reliably shows a steady state extensional flow is that in Fig. 7(b), which was taken using the filament stretching rheometer of the Hassager group $[35,45]$.

\section{Note on the CCR parameter $\boldsymbol{\beta}_{\mathrm{CCR}}$}

In the original paper presenting the Rolie-Poly model [10], the CCR parameter $\beta_{\mathrm{CCR}}$ was conceptually introduced as a universal constant for the rheology of linear entangled polymers, independent of chemistry and molecular weight. It was found that $\beta_{\mathrm{CCR}}=1$ was the smallest possible value that would prevent excessive shear thinning (i.e., shear banding) for all possible polymers at all possible shear rates. Therefore, the authors recommended $\beta_{\mathrm{CCR}}=1$ as a default value of the parameter. At the same time, however, the authors recognized that for the purposes of quantitative comparisons to experimental data at modest entanglement numbers, $Z \sim 20$, lower values of $\beta_{\mathrm{CCR}}$ lead to better results.
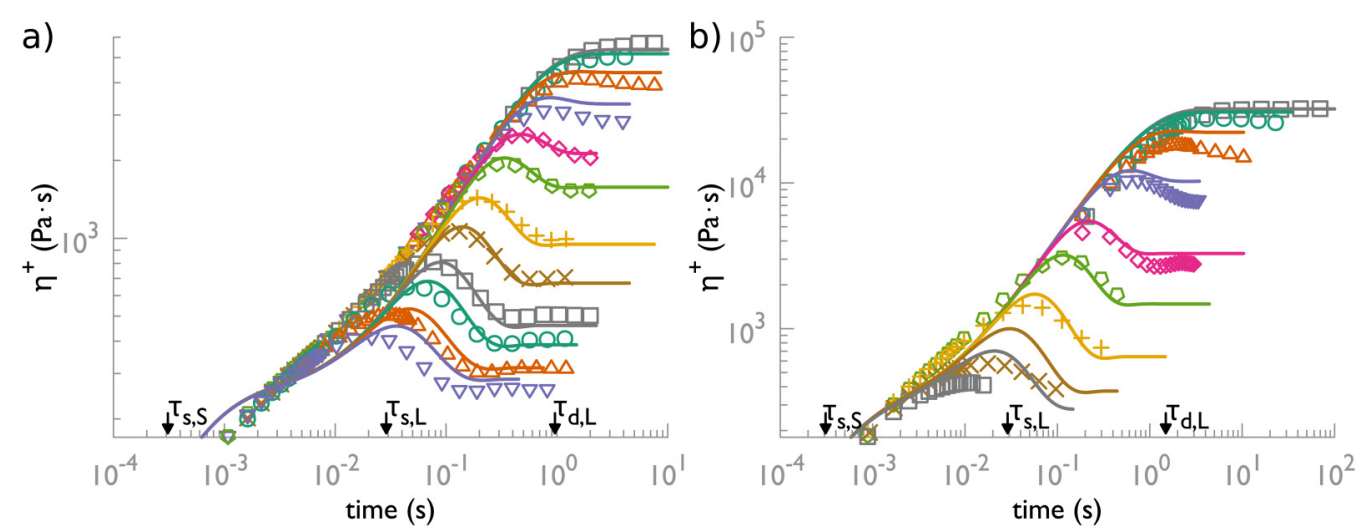

FIG. 8. (a) and (b) Model predictions using $\beta_{\mathrm{CCR}}=0$ to be compared with the model predictions in shear of Figs. 4(b) and 5(b), respectively. 
In this regard, there is precedent to treat $\beta_{\mathrm{CCR}}$ as a fitting parameter rather than a universal rheological constant. In the context of our blend models, allowing $\beta_{\mathrm{CCR}}$ to vary but fixing $\beta_{\text {th }}=1$ creates a conceptual disconnect with the treatment of CR, but it also offers an indisputably useful handle for tuning the nonlinear rheological predictions of the model. In Fig. 8, we present the model predictions using $\beta_{\mathrm{CCR}}=0$ for the two sets of nonlinear shear data, Figs. 4(b) and 5(b). For this particular case, it is evident that using $\beta_{\mathrm{CCR}}=0$ instead of $\beta_{\mathrm{CCR}}=1$ improves the model predictions at high shear rates. Note that the $\beta_{\mathrm{CCR}}$ parameter has practically no influence on the model predictions for elongational flow. Hence, in Sec. III, focusing on elongational data of polydisperse polymer melts, the value we use $\left(\beta_{\mathrm{CCR}}=1\right)$ is rather unimportant.

\section{POLYDISPERSE MELTS: BLEND OF $\boldsymbol{n}$ SPECIES}

\section{A. Generalization of the RDP and SRDP models to $n$ species}

We can generalize Eqs. (12)-(15) to a blend of $n$ monodisperse entangled linear polymers. The total stress is then the sum of the stresses coming from each species $i$, weighted by their volume fraction, $\phi_{i}$, and finite extensibility function related to their stretch $\lambda_{i}$

$$
\boldsymbol{\sigma}=G_{N}^{0} \sum_{i=1}^{n} \phi_{i} \mathrm{f}_{\mathrm{E}}\left(\lambda_{i}\right) \boldsymbol{A}_{i} \quad \text { with } \quad \lambda_{i}=\left(\operatorname{Tr} \boldsymbol{A}_{i} / 3\right)^{1 / 2}
$$

The conformation tensor $\boldsymbol{A}_{i}$ accounts for the stresses that come from the interaction of the species $i$ with itself and the other $(n-1)$ species. Hence, we write

$$
\boldsymbol{A}_{i}=\sum_{j=1}^{n} \phi_{j} \boldsymbol{A}_{i j}
$$

where $\boldsymbol{A}_{i j}$ is the stress conformation tensor on the $i$-chains coming from their entanglements with the $j$-chains.

$$
\begin{aligned}
\nabla_{i j}= & -\frac{1}{\tau_{\mathrm{d}, i}}\left(\boldsymbol{A}_{i j}-\boldsymbol{I}\right)-\frac{2\left(1-\lambda_{i}^{-1}\right)}{\tau_{\mathrm{s}, i}} \mathrm{f}_{\mathrm{E}}\left(\lambda_{i}\right) \boldsymbol{A}_{i j} \\
& -\left(\boldsymbol{A}_{i j}-\boldsymbol{I}\right)\left(\frac{\beta_{\mathrm{th}}}{\tau_{\mathrm{d}, j}}+\beta_{\mathrm{CCR}} \frac{2\left(1-\lambda_{j}^{-1}\right)}{\tau_{\mathrm{s}, j}} \mathrm{f}_{\mathrm{E}}\left(\lambda_{j}\right) \lambda_{i}^{2 \delta}\right) .
\end{aligned}
$$

Equations (45)-(47) constitute the polydisperse RDP model. Similarly, Eqs. (45), (46), and (48) define the corresponding polydisperse SRDP model:

$$
\begin{aligned}
\stackrel{\nabla}{A}_{i j}= & -\frac{1}{\tau_{\mathrm{d}, i}}\left(\boldsymbol{A}_{i j}-\boldsymbol{I}\right)-\frac{1}{2}\left(\frac{2\left(1-\lambda_{i}^{-1}\right)}{\tau_{\mathrm{s}, i}} \mathrm{f}_{\mathrm{E}}\left(\lambda_{i}\right)+\frac{2\left(1-\lambda_{j}^{-1}\right)}{\tau_{\mathrm{s}, j}} \mathrm{f}_{\mathrm{E}}\left(\lambda_{j}\right)\right) \boldsymbol{A}_{i j} \\
& -\left(\boldsymbol{A}_{i j}-\boldsymbol{I}\right)\left(\frac{\beta_{\mathrm{th}}}{\tau_{\mathrm{d}, j}}+\frac{1}{2} \beta_{\mathrm{CCR}}\left(\frac{2\left(1-\lambda_{i}^{-1}\right)}{\tau_{\mathrm{s}, i}} \mathrm{f}_{\mathrm{E}}\left(\lambda_{i}\right) \lambda_{i}^{2 \delta}+\frac{2\left(1-\lambda_{j}^{-1}\right)}{\tau_{\mathrm{s}, j}} \mathrm{f}_{\mathrm{E}}\left(\lambda_{j}\right) \lambda_{j}^{2 \delta}\right)\right)
\end{aligned}
$$

Given that these two polydisperse models are also in quantitative agreement for the predictions that follow, we focus again on the RDP model results. To compute rheological predictions from the model, we only require information about the molecular weight distribution and the tube model parameters $\left(M_{\mathrm{e}}, \tau_{\mathrm{e}}\right.$, and $\left.G_{N}^{0}\right)$ of the material, from which we can obtain the volume fractions $\phi_{i}$ and reptation and stretch relaxation times, $\tau_{\mathrm{d}, i}$ and $\tau_{\mathrm{s}, i}$, respectively.

For the case of bidisperse blends, as discussed in Sec. II D 3 , the qualitative features of the data were well predicted by simple estimates of the relaxation times and volume fraction of long chains [see dashed lines in Figs. 4(b)-7(b)]. However, quantitatively matching the data (especially, the linear rheology) required more detailed calculation, and was significantly improved by treating the volume fraction $\tilde{\phi}_{\mathrm{L}}$ as an adjustable parameter (though constrained to a physically meaningful range $\tilde{\phi}_{\mathrm{L}}<\phi_{\mathrm{L}}$ ). Similar considerations apply for polydisperse blends. As we will show below, it is again possible to capture the qualitative features of the data via simple estimates of relaxation times and weight of each fraction: If the goal of the modeling exercise is to investigate these qualitative effects of polydispersity then this is sufficient. However, quantitative predictions of experimental data require more work in order to obtain the relaxation times and contribution to the modulus of each component in the polydisperse mixture. We have found that the broad distribution of relaxation times in truly polydisperse mixtures means that a double reptation approximation can result in successful quantitative predictions, provided care is taken in computing the appropriate CLF corrections for each $\tau_{\mathrm{d}, i}$, as we discuss in the Appendix. In Sec. III B, we present the RDP model predictions with and, for comparison, without the CLF corrections presented in Subsection 2 of the Appendix.

\section{B. Comparison with experimental data}

We compare our RDP model predictions with elongational viscosity data of three sets of polydisperse polystyrene (PS) melts from [46-48]. If the molecular weight distribution contains short unentangled chains, we renormalize each volume fraction using Eq. (A1), and the literature values of the entanglement molecular weight, entanglement relaxation time, and plateau modulus using Eqs. (A2)-(A4), respectively. The stretch relaxation times are calculated as $\tau_{\mathrm{s}, i}=Z_{i}^{2} \tau_{\mathrm{e}}$. There are then two options: (i) we may use the "naïve" relation $\tau_{\mathrm{d}}=$ 

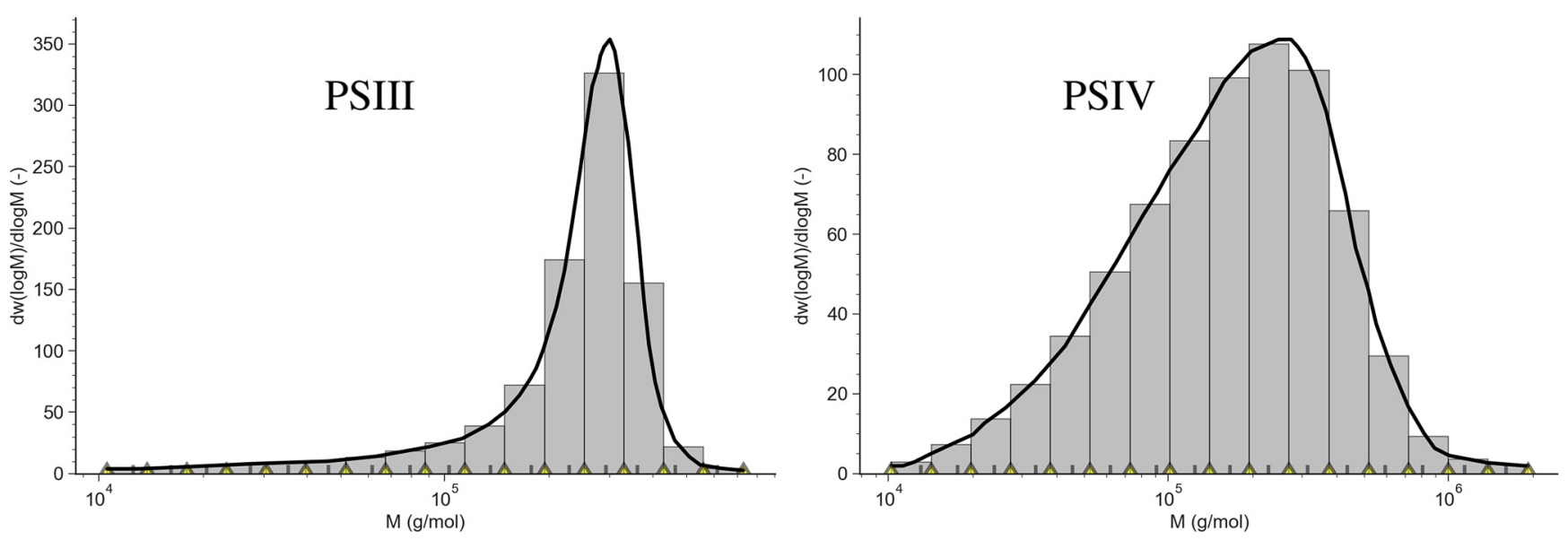

FIG. 9. Molar mass distribution of the PS III and PS IV blends of [46] (lines) and discretization (bars) produced by RepTate software. The area of the ith bar represents $\phi_{i}$, and its associated molecular mass, $M_{i}$, is the weight-averaged molecular mass of the bar width, represented by the thick tick marks. Characteristics of these distributions are reported in Table IV, and the discretization method is discussed in the main text.

$3 Z^{3} \tau_{\mathrm{e}}$ to calculate the $\tau_{\mathrm{d}, i}$ or (ii) we make predictions of the reptation relaxation times using the "recipe" suggested in Eqs. (A5)-(A7) and include the CLF corrections to the modulus; this involves calculating an effective dilution factor $\phi_{\text {dil }}$ for the dominant CLF mode of each component. These two options result in the dashed and solid lines, respectively, in Figs. 10, 12(a), and 13.

We remind the reader that the polydisperse RDP model, Eqs. (45)-(47), has no free fitting parameters to predict the linear and nonlinear rheology of polydisperse melts: The model only requires the volume fractions $\phi_{i}$ and reptation and stretch relaxation times, $\tau_{\mathrm{d}, i}$ and $\tau_{\mathrm{s}, i}$, which can be obtained from the molecular weight distribution and tube model parameters $\left(\tau_{\mathrm{e}}^{0}, M_{\mathrm{e}}^{0}, \tilde{G}_{N}^{0}\right)$ of the material, according to the Appendix. Note that the same tube model parameters are used in the model for the three PS samples presented below.

We also note that the polydisperse RDP model with all the corrections aiming at improving the quantitative model predictions, as presented in the Appendix, has now been added to the new version of the free and open source rheology software RepTate, ${ }^{3}$ developed by some of the authors of this manuscript. Additionally, RepTate software presents a molecular weight distribution discretization module (described below) from which the RDP model can predict a corresponding set of stress relaxation times, as presented in the Appendix.

\section{1. "PS III and PS IV" of Münstedt}

We duplicate the molar mass distribution of two polydisperse PS samples, presented in Fig. 1 of [46], using WebPlotDigitizer, ${ }^{4}$ a web based tool to extract data from plots and images. The result are the black lines in Fig. 9. We discretize the molar mass distributions of these two PS

\footnotetext{
${ }^{3}$ Ramirez, J. and V. A. H. Boudara, "RepTate software," (2018). Documentation and installation instructions: http://reptate.readthedocs.io/. ${ }^{4}$ https://automeris.io/WebPlotDigitizer/.
}

samples using the "discretize" theory of RepTate. ${ }^{3}$ The results of the discretization are the bar plots in Fig. 9, where

(i) The bin edges are equally spaced in log-scale (by default).

(ii) The area of the $i$ th bin (height times width in log-scale) is equal to the area under the curve delimited by the bin edges. It represents the volume fraction $\phi_{i}^{0}$ used in our model [see Eq. (A1)].

(iii) The molecular mass, $M_{i}$, is the weight-averaged molecular mass calculated over the bin width. It is represented by the thick tick mark on the $x$-axis.

We used 16 discretization bins for both the PS III and PS IV samples and verified that a higher number (two and four times more) does not change the subsequent nonlinear rheology predictions.

The characteristics of the two PS samples, PS III and PS IV, are tabulated in Table IV, where

$$
\frac{\left\langle M^{k}\right\rangle}{M^{k}} \equiv \frac{\sum_{i} \phi_{i}^{0} M_{i}^{k}}{\left(\sum_{i} \phi_{i}^{0} M_{i}\right)^{k}}, \quad k \geq 2
$$

are high-order molar mass averages that emphasize the high molar mass tail of the molar mass distribution. We can see from Table IV that while the PS III sample has a higher weight-average molar mass, $M_{w}$, than the PS IV sample, it has a smaller polydispersity, $M_{w} / M_{n}$, and a shorter high-molar mass tail. All experimental data were collected at $160{ }^{\circ} \mathrm{C}$, and PS literature values for the entanglement parameters are $\tau_{\mathrm{e}}^{0}=3.4 \times 10^{-3} \mathrm{~s},{ }^{5} \quad M_{\mathrm{e}}{ }^{0}=16.6 \mathrm{~kg} / \mathrm{mol}$, and $\tilde{G}_{N}^{0}=0.2 \mathrm{MPa}$ [49].

Figure 10 presents the predicted Hencky stress growth coefficient (elongational viscosity), for the PS III and PS IV samples at various elongation rates. We may note that the linear rheology is not perfectly predicted: The model slightly over predicts the LVE for the PS IV blend. The discrepancies in the LVE between the experimental data and the model

\footnotetext{
${ }^{5}$ Material database of RepTate software reptate.com.
} 
TABLE IV. Characteristics of the two PS melts. "Ref." and "Discr." are values reported in [46] and calculated from the bin representation of Fig. 9 (used to produce the predictions of Fig. 10), respectively.

\begin{tabular}{lccccc}
\hline \hline & \multicolumn{2}{c}{ PS III } & & \multicolumn{2}{c}{ PS IV } \\
\cline { 2 - 3 } \cline { 5 - 6 } & Ref. & Discr. & & Ref. & Discr. \\
\hline$M_{w}(\mathrm{~kg} / \mathrm{mol})$ & 253 & 253 & & 219 & 224 \\
$M_{w} / M_{n}$ & 1.9 & 1.56 & & 2.3 & 2.07 \\
$\left\langle M^{2}\right\rangle / M^{2}$ & 1.19 & 1.17 & & 1.69 & 1.70 \\
$\left\langle M^{3}\right\rangle / M^{3}$ & 1.55 & 1.51 & & 4.2 & 4.32 \\
$\left\langle M^{4}\right\rangle / M^{4}$ & 2.23 & 2.09 & & 14.55 & 15.0 \\
\hline \hline
\end{tabular}

indicates that more work on the prediction of the $\tau_{\mathrm{d}, i}$ is required for quantitatively accurate predictions of linear rheology. Our focus, however, is on the nonlinear rheology, and we can see that despite the relatively small differences between the molar mass distribution of the PS III and PS IV samples, cf. Fig. 9 and Table IV, there is an obvious difference in their response to nonlinear flow. This difference is captured by our model, especially the elongation hardening feature, i.e., where the viscosity departs from the LVE, as we now discuss.

Experimental data of the PS IV sample showed a clear elongation hardening at the two highest elongation rates, $\dot{\varepsilon}=\left\{0.07,0.3 \mathrm{~s} \mathrm{~s}^{-1}\right.$, see Fig. 10 , and marginally at $\dot{\varepsilon}=0.015 \mathrm{~s}^{-1}$. These features are captured by the RDP model, which shows elongation hardening occurring at the two highest elongation rates, $\dot{\varepsilon}=\{0.07,0.3\} \mathrm{s}^{-1}$. In contrast, our RDP model predicts that the PS III sample does not present elongation hardening, even at the highest flow rate, $\dot{\gamma}=0.07 \mathrm{~s}^{-1}$. These results are in qualitative agreement, for the elongation hardening feature, with experimental data of Munstedt [46], where the PS III sample does not present elongation hardening at $\dot{\varepsilon}=0.07 \mathrm{~s}^{-1}$, while the stress response of the PS IV sample is above the LVE at that flow rate. Indeed, it is clear that even when the "naïve" expression for the $\tau_{\mathrm{d}, i}$ is used [Eq. (41), thin dashed lines in Fig. 10], then the qualitative features

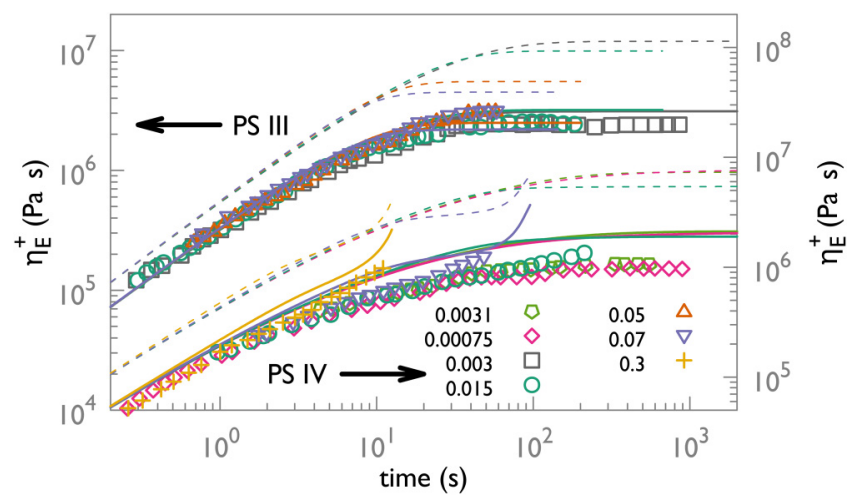

FIG. 10. Logarithm of the elongational viscosity as a function of time for the two PS blends described in Fig. 9. Top curves: PS III with $\dot{\varepsilon}=\{0.003,0.015,0.05,0.07\} \mathrm{s}^{-1}$. Bottom curves: PS IV with $\dot{\varepsilon}=$ $\{0.00075,0.0031,0.015,0.07,0.3\}^{-1}$. Symbols are the experimental observations. The dashed and solid lines are the RDP model predictions using, for $\tau_{\mathrm{d}, i}$, Eq. (41) and the CLF corrections presented in Section 2 of the Appendix, respectively. Tube model parameters are $\tau_{\mathrm{e}}^{0}=3.4 \times 10^{-3} \mathrm{~s}$, $M_{\mathrm{e}}{ }^{0}=16.6 \mathrm{~kg} / \mathrm{mol}$, and $\tilde{G}_{N}^{0}=0.2 \mathrm{MPa}$. pertaining to extension hardening are captured, even if the LVE is not.

\section{2. "Spiked PS" of Minegishi et al.}

We compare our RDP model predictions with experimental data of a polydisperse PS sample $\left(M_{w}=4.23 \times 10^{5} \mathrm{~g} / \mathrm{mol}\right.$, $\left.M_{w} / M_{n}=2.36\right)$ blended with $1.5 \mathrm{wt}$. \% of ultra-high molecular weight PS $\left(M_{w}=3.22 \times 10^{6} \mathrm{~g} / \mathrm{mol}, M_{w} / M_{n}=1.05\right)$ [47]. The discretization of the molecular weight distribution of that spiked PS sample can be found in [23]. Note that [23] makes good predictions of these data using an alternative nonlinear model. All experimental data were collected at $160{ }^{\circ} \mathrm{C}$, so we use the same PS entanglement parameters as above.

In Fig. 11, we compare the relaxation times we obtained using Eq. (A5) to those displayed in Table 1 of [23], which are calculated orientation and stretch relaxation times for the theory presented there. The values of $\tau_{\mathrm{s}}$ we obtained are consistently above those reported in [23]. Moreover, the values of $\tau_{\mathrm{d}}$ we calculated do not follow a straight line on the log-log plot as is the case with the values of [23] [which were obtained using the simple relationship $\left.\tau_{\mathrm{d}}(M)=K M^{3.4}\right]$. This is due to the fact that our calculated effective dilution, $\phi_{\text {dil }}$, (see Subsection 2 of the Appendix for details) does not decrease linearly with increasing chain molecular weight: The speed-up in relaxation is more pronounced for the long chains. Note that, rather than the "continuous" molecular weight distribution (as was the case for the Münstedt samples above), only the "alreadydiscretized" set of volume fractions and molecular mass components was published.

In Fig. 12, we present the model predictions of the elongational viscosity corresponding to the two sets of relaxation times presented in Fig. 11. In Fig. 12(a), we use the reptation and stretch relaxation times calculated using Eq. (A5). In Fig. 12(b), we use the reptation and stretch relaxation times directly extracted from Table 1 of [23].

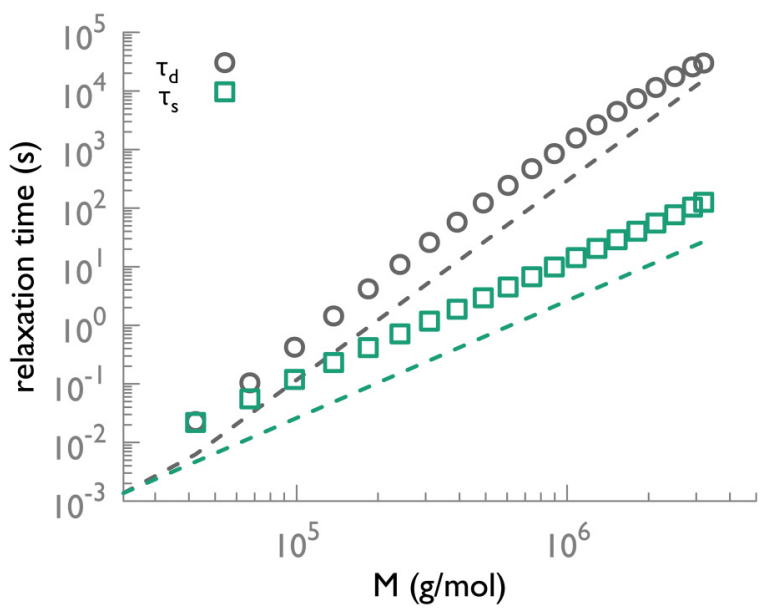

FIG. 11. Orientation and stretch relaxation times used in our simulations. Symbols are the relaxation times calculated from the molecular weight distribution using Eq. (A5). Dashed lines are the relaxation times given in Table 1 of [23]. The corresponding model predictions of the viscosity are presented in Figs. 12(a), and 12(b), respectively. 

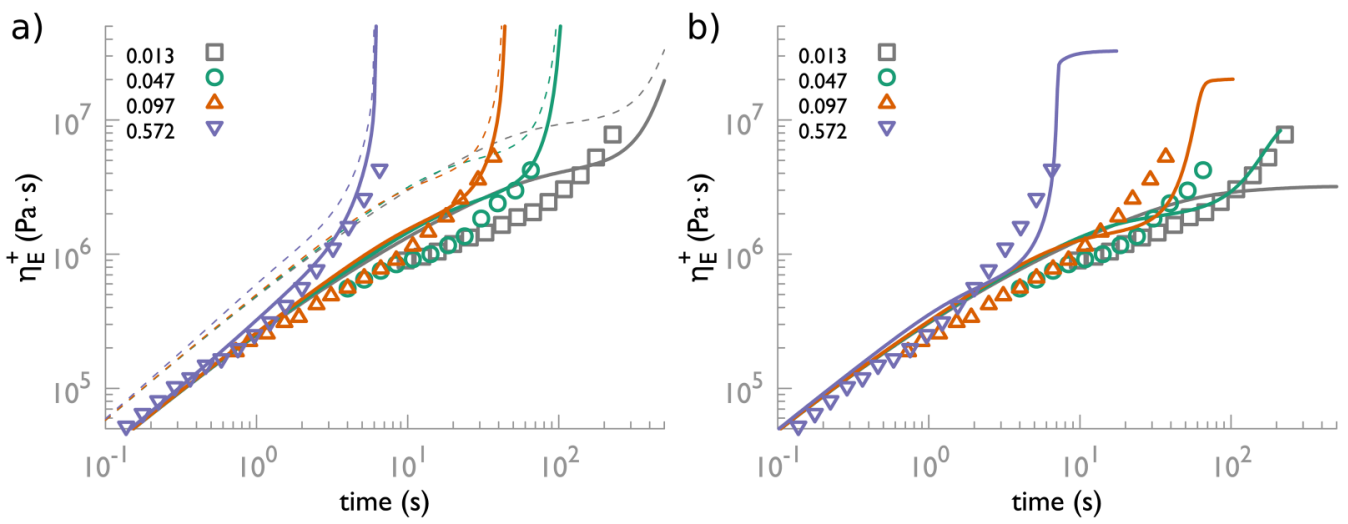

FIG. 12. Extensional viscosity as a function of time for the spiked PS sample for $\dot{\varepsilon}=\{0.013,0.047,0.097,0.572\} \mathrm{s}^{-1}$, as indicated in the figure legend. (a) With relaxation times calculated from the molecular weight distribution using Eq. (A5). (b) Using the relaxation times from Table 1 of [23]. Symbols are the experimental observations. The dashed and solid lines are the RDP model predictions using, for $\tau_{\mathrm{d}, i}$, Eq. (41) and the CLF corrections presented in Section 2 of the Appendix, respectively. Tube model parameters are $\tau_{\mathrm{e}}^{0}=3.4 \times 10^{-3} \mathrm{~s}, M_{\mathrm{e}}{ }^{0}=16.6 \mathrm{~kg} / \mathrm{mol}$, and $\tilde{G}_{N}^{0}=2 \times 10^{5} \mathrm{~Pa}$.

We note that the LVE is better captured by the relaxation times used in Fig. 12(b), which were generated using the empirical relationship $\tau_{\mathrm{d}}(M)=K M^{3.4}$. However, we note that the elongation hardening feature is better captured in Fig. 12(a), i.e., using the RDP model with our calculated relaxation times rather than the ones from Table 1 of [23]. In particular, the experimental data show elongation hardening at all rates, which is clearly captured in Fig. 12(a) for the three highest elongation rate, and a milder hardening for the lowest one, whereas, in Fig. 12(b), elongation hardening in only seen for the two highest elongation rates. This observation supports our CLF corrections to reptation time (Appendix) grounded in detailed physics rather than empirical correlations.

\section{3. "P1" solution of Ye and Sridhar}

We compare our RDP model prediction against a 7\% solution of entangled polydisperse PS with an average molecular weight of $2.65 \times 10^{6}$, which $\mathrm{Ye}$ and Sridhar [48]

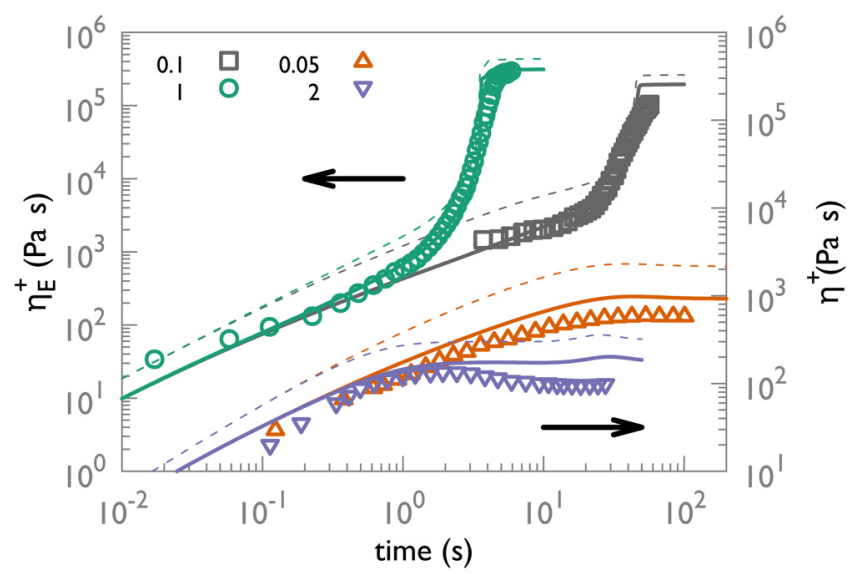

FIG. 13. Uniaxial elongation $\left(\dot{\varepsilon}=\{0.1,1\} \mathrm{s}^{-1}\right)$ and shear $(\dot{\gamma}=\{0.05,2\}$ $\mathrm{s}^{-1}$ ) rheology of the P1 sample of [48], top and bottom curves, respectively. Symbols are the experimental observations. The dashed and solid lines are the RDP model predictions using, for $\tau_{\mathrm{d}, i}$, Eq. (41) and the CLF corrections presented in Section 2 of the Appendix, respectively. Tube model parameters are $\tau_{\mathrm{e}}^{0}=5 \times 10^{-3} \mathrm{~s}, M_{\mathrm{e}}^{0}=235 \mathrm{~kg} / \mathrm{mol}$, and $\tilde{G}_{N}^{0}=619 \mathrm{~Pa}[48]$. produced by mixing 18 components of nearly monodisperse PS ranging from $5 \times 10^{4}$ to $3 \times 10^{7} \mathrm{~g} / \mathrm{mol}$. We consider each molecular mass component and their respective weight fractions as input for our model, similarly to the "spiked PS" case above. Experimental data were collected at $21^{\circ} \mathrm{C}$. We use $\tau_{\mathrm{e}}^{0}=5 \times 10^{-3} \mathrm{~s}, M_{\mathrm{e}}^{0}=235 \mathrm{~kg} / \mathrm{mol}$, and $\tilde{G}_{N}^{0}=619 \mathrm{~Pa}$ which are the entanglement molecular mass and plateau modulus of a 7\% solution of PS, as reported by [48].

In Fig. 13, we present the experimental viscosity at various flow rates, both in shear (bottom symbols) and uniaxial elongation (top symbols), reported in [48], together with our RDP model predictions. The RDP model provides a good quantitative prediction in uniaxial elongation and captures the onset of elongation hardening. In shear, we observe that the model predictions are slightly above the LVE and the steady state viscosity is also slightly over-predicted. However, the qualitative trend is similar to the experimental data for both shear rates. The model predictions corresponding to $\dot{\gamma}=2$ exhibit a small shoulder at late time. Upon close inspection of the experimental viscosity data, a nearly undetectable increase in viscosity at very late time can be observed too.

\section{CONCLUSIONS}

In this work, we proposed two simplified tube models for polydisperse melts of linear polymers which combined the success of the double reptation approximation [15] in the linear regime with the success of the Rolie-Poly constitutive equation [10] in the nonlinear regime. These two models have different origins: The RDP model is simplified from the Read et al. model [26], while the SRDP model combines the same building blocks but in a symmetric fashion for a computational advantage. Overall, however, the RDP and SRDP model predictions were found to be effectively indistinguishable from one another in the cases presented in this work (and for a wide set of parameters). In the case of bidisperse blends of long and short chains, we showed that the RDP model contained the "enhanced stretch relaxation time" that was observed experimentally [9] and explained theoretically [26]. Given the quantitative similarity in the predictions for 
the two models, we show only the RDP model predictions and comparisons with experimental data for the case of bidisperse blends, for various materials, in the linear rheology regime and in both nonlinear shear and uniaxial extension. The RDP and SRDP models require no fitting parameters for qualitative purposes, but for quantitative interests we allow a single fitting parameter, constrained to a narrow interval, to correct for departures from the double reptation's binary view of entanglements in the bidisperse case. It is adjusted using linear rheology data and the nonlinear rheology predictions are subsequently produced without extra fitting parameters. The model quantitatively matched the experimental data in elongation at all elongation rates. This means that we are capturing enhanced stretch relaxation time correctly. In shear, the model was less successful at predicting the blend's rheology at moderate to high shear rates, where the more detailed model of Read et al. [26] had no difficulty by comparison. Here, we found it was possible to improve quantitative agreement with an individual experimental data set by empirically adjusting the value of the CCR parameter, $\beta_{\mathrm{CCR}}$, as has previously been done for matching experimental data in monodisperse melts.

Finally, we generalized the RDP and SRDP models to a blend of $n$ monodisperse entangled linear polymers and developed a method to account for the CLF effects on the reptation times. This method, aimed at improving the qualitative fit to data, corrects the naïve " $\tau_{\mathrm{d}}=3 Z^{3} \tau_{\mathrm{e}}$ " estimate and proved to be robust when we compared the elongational flow predictions of the RDP model to experimental nonlinear rheology observation of the polydisperse "PS III" and "PS IV" of [46], "spiked PS" of [47], and "P1 solution" of [48]. However, we observed that our method for determining relaxation times and moduli, detailed in the Appendix, has a general tendency to (slightly) overpredict the LVE.

Considering the PS IV sample, which contained high molar-mass components, the elongation hardening that was seen experimentally in [46] could be qualitatively reproduced using our RDP model: It showed elongation hardening occurring at the same elongation rates as the experimental data. In contrast, the "PS III" sample, which did not contain high molar-mass components, did not present the hardening features experimentally, and this was also reproduced correctly by our RDP model. Then, we considered the "spiked PS," a polydisperse PS sample blended with a high molecular weight component [47]. The RDP model captured successfully the extension hardening at all rates. Finally, the RDP model predictions showed qualitative, in shear to quantitative, in elongation, match to the experimental data of the "P1" polydisperse solution of entangled PS of [48].

Hence, given a molecular mass distribution, the RDP and SRDP models can successfully predict when, and at which elongation rate, elongation hardening might occur. We emphasize that the models do so without any fitting parameter.

We also note that the RDP model, with the static and dynamic dilution corrections presented in this work, is readily available in the free and open source RepTate software, ${ }^{3}$ where the user can make predictions of start-up shear and elongation rheology with the molecular weight distribution of the polydisperse polymeric material as input.
The work presented here is therefore a step forward to the possibility of predicting the rheological properties of industrially polydisperse polymer melts accurately and robustly, which would be a major advance for industries manufacturing textiles, automobiles, aircraft, paints and coatings, cosmetics and consumer products, oil-field fluids, and others. To further demonstrate this final assertion, recent CFD flow simulations using the bidisperse SRDP model will be presented in a forthcoming paper.

\section{ACKNOWLEDGMENTS}

V.A.H.B. and D.J.R. have received funding from the People Programme (Marie Skłodowska-Curie Actions) of the European Union's Seventh Framework Programme (No. FP7/2007-2013) under REA Grant Agreement No. 607937SUPOLEN project. D.J.R. also acknowledges funding from EPSRC Grant No. EP/P005403/1. The participation of L.G.L and J.D.P was supported by funding from the National Science Foundation (No. CBET-1510333). The authors thank Chinmay Das and Tom McLeish for very helpful discussions at many points of this work and insightful comments and suggestions.

\section{APPENDIX: STATIC AND DYNAMIC DILUTION CORRECTIONS}

\section{Static dilution: Short unentangled chains}

In this section, we outline a set of corrections (including the aforementioned CLF corrections) that reflect our present understanding of the detailed physics relevant to entangled polymer blends. Making use of this discussion of detailed physics, in the main body of the paper, we present a quantitative comparison of our parameter-free model, described by Eqs. (45)-(47) against experimentally reported findings on the elongational viscosity of various PS melts [46-48] in Sec. III B.

A well entangled polymer melt with a broad distribution of molecular weights will invariably include some fraction of chains whose molecular weight, $M$, falls below the entanglement molecular weight $M_{\mathrm{e}}^{0}$. It is not appropriate to describe those unentangled chains with the same physics that apply to the larger, well-entangled chains, so we partition the molecular weight distribution into entangled $\left(M>2 M_{\mathrm{e}}^{0}\right)$ and unentangled $\left(M<2 M_{\mathrm{e}}^{0}\right)$ fractions, where, for reasons that will become evident shortly, we denote the entanglement molecular weight in the melt state as $M_{\mathrm{e}}^{0}$. The unentangled fraction, comprising a total volume fraction $\phi_{\mathrm{u}}$, is treated as a smallmolecule solvent and is otherwise neglected from the calculations. We assume that the molecular weight distribution of the entangled fraction is discretized into components, $i$, and renormalize. Specifically, we renormalize (i) the volume fraction of each entangled component so that the total volume fraction of entangled species is unity, (ii) the entanglement molecular weight of the entangled components, (iii) the relaxation time of an entanglement segment, and (iv) the plateau modulus according to

$$
\phi_{i}=\phi_{i}^{0} /\left(1-\phi_{\mathrm{u}}\right)
$$




$$
\begin{gathered}
M_{\mathrm{e}}=M_{\mathrm{e}}^{0} /\left(1-\phi_{\mathrm{u}}\right), \\
\tau_{\mathrm{e}}=\tau_{\mathrm{e}}^{0} /\left(1-\phi_{\mathrm{u}}\right)^{2}, \\
G_{N}^{0}=\left(1-\phi_{\mathrm{u}}\right)^{2} \tilde{G}_{N}^{0},
\end{gathered}
$$

with $\phi_{i}^{0}$ the volume fraction of species $i$ before renormalization, and $M_{\mathrm{e}}^{0}, \tau_{\mathrm{e}}^{0}$, and $\tilde{G}_{N}^{0}$ the literature values for the entanglement molecular weight, entanglement relaxation time, and plateau modulus, respectively. These transformations leave the stretch relaxation times unchanged but decrease the reptation times by a factor $\left(1-\phi_{\mathrm{u}}\right)$. In the following calculations, and in the main text, we use the renormalized quantities.

\section{Dynamic dilution}

This section presents the CLF corrections to the RDP model that one can include in order to gain a more qualitative description of the data. We advise the reader that it is possible to ignore these corrections (and return to Sec. III B) by using Eq. (41) for the reptation time of each molecular weight component of the system. In that case, the RDP model produces a good qualitative description of the polydisperse data, as shown by the thin dashed lines in Figs. 10, 12(a), and 13 .

\section{a. CLF corrections to reptation times}

For each molecular mass component, $M_{i}$, of the molecular weight distribution, we approximate the reptation time, $\tau_{\mathrm{d}, i}$, and stretch relaxation time $\tau_{\mathrm{s}, i}$, from the entanglement relaxation time as

$$
\tau_{\mathrm{d}, i}=3 Z_{i}^{3} \mathrm{f}_{\mu}\left(\tilde{Z}_{i}\right) \tau_{\mathrm{e}}, \quad \tau_{\mathrm{s}, i}=Z_{i}^{2} \tau_{\mathrm{e}},
$$

with $Z_{i}=M_{i} / M_{\mathrm{e}}$ being the entanglement number of the $i$ th species, $\mathrm{f}_{\mu}(Z)$ is the CLF correction to reptation as in Eq. (43) [37], and $\tilde{Z}_{i}=\phi_{\mathrm{dil}, i} Z_{i}$ is the effective number of entanglements on the chain $i$, assuming a dilution exponent of 1 [29], where $\phi_{\mathrm{dil}, i}$ is the effective dilution to be calculated below.

Equation (A5) works, broadly, within the "dynamic dilution" picture of tube dynamics, which considers that the effective "tube" constraint felt by a given test chain depends on the time scale of the motion being considered. After relaxation of shorter (entangled) chains, they release their entanglements on longer chains and so, in some sense, act as "solvent" for the longer chains. Hence, depending on the time scale, a test chain may "feel" different tubes, as illustrated in Fig. 14, with different tubes representing the dilution as various short chain species relax.

However, it is pointed out by Read and co-workers $[1,16,26]$ for the case of binary blends that the relevant tube depends upon the motion being considered. Reptation motion along the contour of a smoother "diluted" tube requires the chain to move a shorter distance, but it involves a larger friction because each step along the diluted tube requires CR from the shorter chains. On the other hand, reptation along the thinnest tube is subject only to the friction from the chain monomers. In most cases, the fastest reptation motion

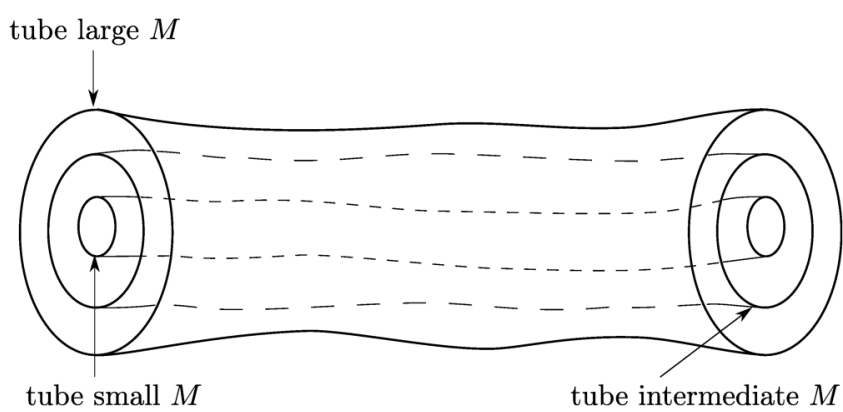

FIG. 14. Sketch of the different tubes within which the components are confined. The components with the highest molar mass $(M)$ has the fattest tube, made of entanglements with its own components only. The components with the smallest molar mass experiences the thinnest tube, made of entanglements with all other components. Intermediate molar mass components are confined somewhere in between.

remains the low-friction motion along the thinnest tube, and this is reflected in Eq. (A5) where it is the bare reptation time for motion along the thin tube $\left(3 Z_{i}^{3} \tau_{\mathrm{e}}\right)$ that is modified by CLF contributions. However, even if reptation is fastest along the thinner tube, the extra freedom given to the chain by $\mathrm{CR}$ events can permit deeper CLFs commensurate with the diluted tube, which speeds up the terminal relaxation because CLF shortens the distance required to reptate [16,26]. This is reflected in the factor $\mathrm{f}_{\mu}(\tilde{Z})$ of Eq. (A5), but we need to make a choice as to which diluted tube is appropriate for the CLF of a given test chain. For a given dilution, $\phi_{\text {dil }}$, the predicted CLF time scale is, according to [26], $\tau_{\mathrm{s}} / \phi_{\text {dil }}$.

For the purposes of the present work, we make the following assumptions: (i) The effective dilution at a given time scale $t$ is $\phi_{\mathrm{dil}}(t)$, which is equal to the sum of the volume fractions of all chains with relaxation time greater than $t$; (ii) CLF makes use of the most diluted tube available at the CLF time scale. Hence, the dilution $\phi_{\text {dil }}$ used in the formula for the effective CLF time $\tau_{\mathrm{s}}^{\text {eff }}=\tau_{\mathrm{s}} / \phi_{\text {dil }}$ is simply the effective dilution at that time scale, i.e., $\phi_{\text {dil }}\left(\tau_{\mathrm{s}}^{\text {eff }}\right)$. This gives a selfconsistent formula which can be solved to obtain the CLF time, and appropriate dilution factor for CLF, for a given chain species. The value of $\tilde{Z}$ for that species is then $\tilde{Z}=\phi_{\mathrm{dil}} Z$. This rule allows a prediction to be made for the relaxation time of each species, and so gives a prediction for the linear rheology.

In practice, given a set of $n$ molecular mass modes, $\left\{M_{i}, \phi_{i}\right\}$, to find the value of the dilution factor, $\phi_{\mathrm{dil}, i}$, for the species $i$, we solve

$$
\left\{\begin{aligned}
\tau_{\mathrm{s}, i}^{\mathrm{eff}} & =\frac{Z_{i}^{2} \tau_{\mathrm{e}}}{\phi_{\mathrm{dil}, i}}, \\
\phi_{\mathrm{dil}, i} & =\sum_{j_{\mathrm{min}, i}}^{n} \phi_{j},
\end{aligned}\right.
$$

with $j_{\min , i}$ the smallest $j$ such that $\tau_{\mathrm{s}, i}^{\text {eff }}<\tau_{\mathrm{d}, j}$, assuming the species are ordered: $\tau_{\mathrm{d}, 1}<\tau_{\mathrm{d}, 2}<\cdots<\tau_{\mathrm{d}, n}$, and $\phi_{j}$ is the volume fraction of the species $j$, as in Eq. (45). In case $\tau_{\mathrm{s}, i}^{\mathrm{eff}}>\tau_{\mathrm{d}, n}$, we set $\phi_{\mathrm{dil}, i}=\phi_{n}$.

We solve Eq. (A6) iteratively, starting with the shortest chains (i.e., $i=1$ ), for which we set $\phi_{\text {dil, } 1}=1$ (no dilution), and the reptation times are computed using Eq. (A5), where the unknown dilution factors, $\phi_{\text {dil }}$, are initially set equal to 1 . 
Due to the discretization of the molar mass distribution, Eq. (A6) gives the same $\phi_{\text {dil }}$ for all species $i$ such that $\tau_{\mathrm{d}, j-1}<\tau_{\mathrm{s}, i}^{\text {eff }}<\tau_{\mathrm{d}, j}$ and so $\phi_{\mathrm{dil}}$ would follow steps as a function of $i$. In practice, we do a linear interpolation to ensure that the dilution factor is "smoothly" decreasing, i.e., we use

$$
\begin{gathered}
\phi_{\mathrm{dil}, i}=\phi_{\mathrm{int}, i}+\sum_{j_{\min , i}}^{n} \phi_{j} \quad \text { where } \\
\phi_{\mathrm{int}, i}=\frac{\tau_{\mathrm{d}, j_{\min , i}}-\tau_{\mathrm{s}, i}^{\mathrm{eff}}}{\tau_{\mathrm{d}, j_{\min , i}}-\tau_{\mathrm{d}, j_{\min , i}-1}} \phi_{j_{\min , i}-1} .
\end{gathered}
$$

The interpolated value, $\phi_{\text {int }, i}$, depends on the "distance" between $\tau_{\mathrm{s}, i}^{\text {eff }}$ and the two neighboring values of $\tau_{\mathrm{d}}$.

In cases where the dilution factor obtained is such that $Z_{i} \phi_{\mathrm{dil}, i}<1$, we conclude that the species $i$ is effectively unentangled so that terminal relaxation is via CR Rouse motion $[1,29,50]$ rather than reptation: We set $\tau_{\mathrm{d}, i}$ to be a solution of $Z_{i} \phi_{\mathrm{dil}, i}\left(\tau_{\mathrm{d}, i}\right)=1$, using Eq. (A7) to do the interpolation of $\phi_{\mathrm{dil}, i}$. This gives the time when the species $i$ becomes unentangled; we take this time to be the terminal time of that species.

\section{b. CLF corrections to the modulus}

Primitive path fluctuations also reduce the terminal modulus because of the shortened tube [1,6], therefore, we correct Eq. (45) by a factor $g_{v}[37,38]$. The total stress is then

$$
\boldsymbol{\sigma}=G_{N}^{0} \sum_{i=1}^{n} g_{v}\left(\tilde{Z}_{i}\right) \phi_{i} \mathrm{f}_{\mathrm{E}}\left(\lambda_{i}\right) \boldsymbol{A}_{i},
$$

with $\tilde{Z}_{i}=Z_{i} \phi_{\text {dil, } i}$ and, according to Doi [38] and Likhtman and McLeish [37]

$$
g_{v}(\tilde{Z})=1-\frac{1.69}{\tilde{Z}^{1 / 2}}+\frac{2.0}{\tilde{Z}}-\frac{1.24}{\tilde{Z}^{3 / 2}} .
$$

\section{References}

[1] Dealy, J. M., D. J. Read, and R. G. Larson, Structure and Rheology of Molten Polymers: From Structure to Flow Behavior and Back Again (Carl Hanser, München, 2018).

[2] Collis, M. W., A. K. Lele, M. R. Mackley, R. S. Graham, D. J. Groves, A. E. Likhtman, T. M. Nicholson, O. G. Harlen, T. C. B. McLeish, L. R. Hutchings, C. M. Fernyhough, and R. N. Young, "Constriction flows of monodisperse linear entangled polymers: Multiscale modeling and flow visualization," J. Rheol. 49, 501-522 (2005).

[3] Hassell, D., J. Embery, T. McLeish, and M. Mackley, “An experimental evaluation of the formation of an instability in monodisperse and polydisperse polystyrenes," J. Non Newton. Fluid Mech. 157, 1-14 (2009).

[4] de Gennes, P.-G., Scaling Concepts in Polymer Physics (Cornell University, Ithaca, 1979).

[5] de Gennes, P.-G., "Reptation of a polymer chain in the presence of fixed obstacles," J. Chem. Phys. 55, 572-579 (1971).

[6] Doi, M., and S. F. Edwards, The Theory of Polymer Dynamics (Oxford University, New York, 1988).

[7] Graham, R. S., A. E. Likhtman, T. C. B. McLeish, and S. T. Milner, "Microscopic theory of linear, entangled polymer chains under rapid deformation including chain stretch and convective constraint release," J. Rheol. 47, 1171-1200 (2003).

[8] Vlassopoulos, D., "Macromolecular topology and rheology: Beyond the tube model," Rheol. Acta 55, 613-632 (2016).
[9] Auhl, D., J. Ramrez, A. E. Likhtman, P. Chambon, and C. Fernyhough, "Linear and nonlinear shear flow behavior of monodisperse polyisoprene melts with a large range of molecular weights," J. Rheol. 52, 801-835 (2008).

[10] Likhtman, A. E., and R. S. Graham, "Simple constitutive equation for linear polymer melts derived from molecular theory: Rolie-Poly equation," J. Non Newton. Fluid Mech. 114, 1-12 (2003).

[11] McIlroy, C., and P. D. Olmsted, "Deformation of an amorphous polymer during the fused-filament-fabrication method for additive manufacturing,” J. Rheol. 61, 379-397 (2017).

[12] McIlroy, C., and P. Olmsted, "Disentanglement effects on welding behaviour of polymer melts during the fused-filament-fabrication method for additive manufacturing," Polymer 123, 376-391 (2017).

[13] Lord, T. D., L. Scelsi, D. G. Hassell, M. R. Mackley, J. Embery, D. Auhl, O. G. Harlen, R. Tenchev, P. K. Jimack, and M. A. Walkley, "The matching of 3D Rolie-Poly viscoelastic numerical simulations with experimental polymer melt flow within a slit and a cross-slot geometry," J. Rheol. 54, 355-373 (2010).

[14] Rubinstein, M., and R. H. Colby, "Self-consistent theory of polydisperse entangled polymers: Linear viscoelasticity of binary blends," J. Chem. Phys. 89, 5291-5306 (1988).

[15] des Cloizeaux, J., "Double reptation vs. Simple reptation in polymer melts," Europhys. Lett. 5, 437-442 (1988).

[16] Read, D. J., M. Shivokhin, and A. E. Likhtman, "Contour length fluctuations and constraint release in entangled polymers: Slip-spring simulations and their implications for binary blend rheology," J. Rheol. 62, 1017-1036 (2018).

[17] Bernstein, B., E. A. Kearsley, and L. J. Zapas, "A study of stress relaxation with finite strain,” Trans. Soc. Rheol. 7, 391-410 (1963).

[18] Ianniruberto, G., and G. Marrucci, "A multi-mode CCR model for entangled polymers with chain stretch," J. Non Newton. Fluid Mech. 102, 383-395 (2002).

[19] Ianniruberto, G., and G. Marrucci, "A simple constitutive equation for entangled polymers with chain stretch,” J. Rheol. 45, 1305-1318 (2001).

[20] Mead, D. W., "Development of the binary interaction theory for entangled polydisperse linear polymers," Rheol. Acta 46, 369-395 (2006).

[21] Mead, D. W., "Analytic derivation of the Cox-Merz rule using the MLD toy model for polydisperse linear polymers," Rheol. Acta 50, 837-866 (2011).

[22] Mead, D., "Small amplitude oscillatory shear flow superposed on parallel or perpendicular (orthogonal) steady shear of polydisperse linear polymers: The MLD model," J. Non Newton. Fluid Mech. 195, 99-113 (2013).

[23] Mishler, S., and D. Mead, "Application of the MLD toy model to extensional flows of broadly polydisperse linear polymers: Part II. Comparison with experimental data," J. Non Newton. Fluid Mech. 197, 80-90 (2013).

[24] Mead, D. W., S. Monjezi, and J. Park, "A constitutive model for entangled polydisperse linear flexible polymers with entanglement dynamics and a configuration dependent friction coefficient. Part I: Model derivation,” J. Rheol. 62, 121-134 (2018).

[25] Wapperom, P., and R. Keunings, "Impact of decoupling approximation between stretch and orientation in rheometrical and complex flow of entangled linear polymers," J. Non Newton. Fluid Mech. 122, 33-43 (2004).

[26] Read, D. J., K. Jagannathan, S. K. Sukumaran, and D. Auhl, "A fullchain constitutive model for bidisperse blends of linear polymers," J. Rheol. 56, 823-873 (2012).

[27] Jupp, L., and X.-F. Yuan, "Dynamic phase separation of a binary polymer liquid with asymmetric composition under rheometric flow," J. Non Newton. Fluid Mech. 124, 93-101 (2004). 
[28] Guo, X.-W., S. Zou, X. Yang, X.-F. Yuan, and M. Wang, "Interface instabilities and chaotic rheological responses in binary polymer mixtures under shear flow," RSC Adv. 4, 61167-61177 (2014).

[29] Viovy, J. L., M. Rubinstein, and R. H. Colby, "Constraint release in polymer melts: Tube reorganization versus tube dilation," Macromolecules 24, 3587-3596 (1991).

[30] Auhl, D., P. Chambon, T. C. B. McLeish, and D. J. Read, "Elongational flow of blends of long and short polymers: Effective stretch relaxation time," Phys. Rev. Lett. 103, 136001 (2009).

[31] Marrucci, G., "Dynamics of entanglements: A nonlinear model consistent with the Cox-Merz rule," J. Non Newton. Fluid Mech. 62, 279-289 (1996).

[32] Kabanemi, K. K., and J. F. Hétu, "Nonequilibrium stretching dynamics of dilute and entangled linear polymers in extensional flow," J. Non Newton. Fluid Mech. 160, 113-121 (2009).

[33] Warner, H. R., "Kinetic theory and rheology of dilute suspensions of finitely extendible dumbbells," Ind. Eng. Chem. Fundam. 11, 379-387 (1972).

[34] Shahid, T., Q. Huang, F. Oosterlinck, C. Clasen, and E. van Ruymbeke, "Dynamic dilution exponent in monodisperse entangled polymer solutions," Soft Matter 13, 269-282 (2017).

[35] Nielsen, J. K., H. K. Rasmussen, O. Hassager, and G. H. McKinley, "Elongational viscosity of monodisperse and bidisperse polystyrene melts," J. Rheol. 50, 453-476 (2006).

[36] Larson, R. G., T. Sridhar, L. G. Leal, G. H. McKinley, A. E. Likhtman, and T. C. B. McLeish, "Definitions of entanglement spacing and time constants in the tube model," J. Rheol. 47, 809-818 (2003).

[37] Likhtman, A. E., and T. C. B. McLeish, "Quantitative theory for linear dynamics of linear entangled polymers," Macromolecules 35, 6332-6343 (2002).

[38] Doi, M., "Explanation for the 3.4-power law for viscosity of polymeric liquids on the basis of the tube model," J. Polym. Sci. B Polym. Phys. 21, 667-684 (1983).

[39] Graessley, W. W., "Some phenomenological consequences of the Doi-Edwards theory of viscoelasticity," J. Polym. Sci. B Polym. Phys. 18, 27-34 (1980).

[40] Fetters, L. J., D. J. Lohse, S. T. Milner, and W. W. Graessley, "Packing length influence in linear polymer melts on the entanglement, critical, and reptation molecular weights," Macromolecules 32, 6847-6851 (1999).
[41] Yaoita, T., T. Isaki, Y. Masubuchi, H. Watanabe, G. Ianniruberto, and G. Marrucci, "Primitive chain network simulation of elongational flows of entangled linear chains: Stretch/orientation-induced reduction of monomeric friction,” Macromolecules 45, 2773-2782 (2012).

[42] Ianniruberto, G., A. Brasiello, and G. Marrucci, "Simulations of fast shear flows of PS oligomers confirm monomeric friction reduction in fast elongational flows of monodisperse PS melts as indicated by rheooptical data," Macromolecules 45, 8058-8066 (2012).

[43] Ianniruberto, G., "Extensional flows of solutions of entangled polymers confirm reduction of friction coefficient," Macromolecules $\mathbf{4 8}$, 6306-6312 (2015).

[44] Masubuchi, Y., Y. Matsumiya, and H. Watanabe, "Test of orientation/ stretch-induced reduction of friction via primitive chain network simulations for polystyrene, polyisoprene, and poly(n-butyl acrylate)," Macromolecules 47, 6768-6775 (2014).

[45] Bach, A., K. Almdal, H. K. Rasmussen, and O. Hassager, "Elongational viscosity of narrow molar mass distribution polystyrene," Macromolecules 36, 5174-5179 (2003).

[46] Münstedt, H., "Dependence of the elongational behavior of polystyrene melts on molecular weight and molecular weight distribution," J. Rheol. 24, 847-867 (1980).

[47] Minegishi, A., A. Nishioka, T. Takahashi, Y. Masubuchi, J.-i. Takimoto, and K. Koyama, "Uniaxial elongational viscosity of PS/ a small amount of UHMW-PS blends," Rheol. Acta 40, 329-338 (2001).

[48] Ye, X., and T. Sridhar, "Effects of the polydispersity on rheological properties of entangled polystyrene solutions," Macromolecules 38, 3442-3449 (2005).

[49] Fetters, L. J., D. J. Lohse, D. Richter, T. A. Witten, and A. Zirkel, "Connection between polymer molecular weight, density, chain dimensions, and melt viscoelastic properties," Macromolecules 27, 4639-4647 (1994).

[50] Milner, S. T., T. C. B. McLeish, R. Young, A. Hakiki, and J. Johnson, "Dynamic dilution, constraint-release, and star-linear blends," Macromolecules 31, 9345-9353 (1998).

[51] See supplementary material at https://doi.org/10.1122/1.5052320 for (i) the link between the model of Read et al. [26] and the RDP model and (ii) a comparison of the RDP and SRDP model predictions. 